\title{
Molecular Topology and the Surface Chemical Bond: Alternant Versus Nonalternant Aromatic Systems as Functional Structural Elements
}

\author{
Benedikt P. Klein, ${ }^{1}$ Nadine J. van der Heijden, ${ }^{2}$ Stefan R. Kachel, ${ }^{1}$ Markus Franke, ${ }^{3}$ Claudio K. Krug,${ }^{1}$ \\ Katharina K. Greulich, ${ }^{1}$ Lukas Ruppenthal, ${ }^{1}$ Philipp Müller, ${ }^{1}$ Phil Rosenow, ${ }^{1}$ Shayan Parhizkar, ${ }^{3}$ \\ François C. Bocquet, ${ }^{3}$ Martin Schmid, ${ }^{1}$ Wolfgang Hieringer, ${ }^{4}$ Reinhard J. Maurer, ${ }^{5}$ Ralf Tonner, ${ }^{1}$ \\ Christian Kumpf, ${ }^{3}$ Ingmar Swart, ${ }^{2}$ and J. Michael Gottfried ${ }^{1, *}$ \\ ${ }^{1}$ Philipps-Universität Marburg, Fachbereich Chemie, Hans-Meerwein-Straße 4, 35032 Marburg, Germany \\ ${ }^{2}$ Condensed Matter and Interfaces, Debye Institute for Nanomaterials Science, \\ Utrecht University, P.O. Box 80000, 3508 TA Utrecht, The Netherlands \\ ${ }^{3}$ Peter Grünberg Institut (PGI-3), Forschungszentrum Jülich, 52425 Jülich, Germany \\ and Jülich Aachen Research Alliance (JARA), Fundamentals of Future Information Technology, \\ 52425 Jülich, Germany \\ ${ }^{4}$ Lehrstuhl für Theoretische Chemie, Universität Erlangen-Nürnberg, \\ Egerlandstraße 3, 91058 Erlangen, Germany \\ ${ }^{5}$ Department of Chemistry and Centre for Scientific Computing, University of Warwick, \\ Gibbet Hill Road, Coventry, CV4 7AL, United Kingdom
}

(Received 15 August 2018; revised manuscript received 22 November 2018; published 13 February 2019)

\begin{abstract}
The interaction of carbon-based aromatic molecules and nanostructures with metals can strongly depend on the topology of their $\pi$-electron systems. This is shown with a model system using the isomers azulene, which has a nonalternant $\pi$ system with a 5-7 ring structure, and naphthalene, which has an alternant $\pi$ system with a 6-6 ring structure. We found that azulene can interact much more strongly with metal surfaces. On copper (111), its zero-coverage desorption energy is $1.86 \mathrm{eV}$, compared to $1.07 \mathrm{eV}$ for naphthalene. The different bond strengths are reflected in the adsorption heights, which are $2.30 \AA$ for azulene and $3.04 \AA$ for naphthalene, as measured by the normal incidence $\mathrm{x}$-ray standing wave technique. These differences in the surface chemical bond are related to the electronic structure of the molecular $\pi$ systems. Azulene has a lowlying LUMO that is close to the Fermi energy of $\mathrm{Cu}$ and strongly hybridizes with electronic states of the surface, as is shown by photoemission, near-edge x-ray absorption fine-structure, and scanning tunneling microscopy data in combination with theoretical analysis. According to density functional theory calculations, electron donation from the surface into the molecular LUMO leads to negative charging and deformation of the adsorbed azulene. Noncontact atomic force microscopy confirms the deformation, while Kelvin probe force microscopy maps show that adsorbed azulene partially retains its in-plane dipole. In contrast, naphthalene experiences only minor adsorption-induced changes of its electronic and geometric structure. Our results indicate that the electronic properties of metal-organic interfaces, as they occur in organic (opto)electronic devices, can be tuned through modifications of the $\pi$ topology of the molecular organic semiconductor, especially by introducing 5-7 ring pairs as functional structural elements.
\end{abstract}

DOI: 10.1103/PhysRevX.9.011030

\section{INTRODUCTION}

Carbon-based materials with aromatic $\pi$-electron systems, such as $\pi$-conjugated molecular solids [1,2], polymers [3,4],

\footnotetext{
*michael.gottfried@ chemie.uni-marburg.de
}

Published by the American Physical Society under the terms of the Creative Commons Attribution 4.0 International license. Further distribution of this work must maintain attribution to the author(s) and the published article's title, journal citation, and DOI.

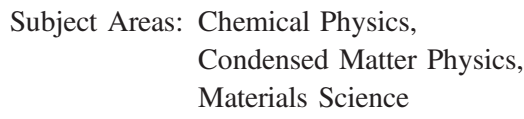

and low-dimensional nanostructures [5,6], have attracted considerable attention as organic semiconductors [7-9]. A crucial aspect for the application of these materials in (opto) electronic devices is their interface formation with metal surfaces at the contacting electrodes. The electronic properties of the resulting metal-organic interfaces determine important performance parameters such as charge carrier injection rates $[10,11]$. Precise control over various interface properties, especially the wave-function overlap and the energy-level alignment, is therefore critical for the rational improvement of organic electronic devices [12]. 
The properties of a $\pi$-electron system are largely determined by its topology, i.e., the connectivity pattern as expressed through the topological matrix in the Hückel molecular orbital (HMO) theory [13]. Most organic semiconductors have carbon backbones with alternant topology because they consists of six-membered rings [1,7]. Only very recently has increased interest in so-called nonalternant aromatic structures [14] arisen in the field of graphene nanoribbons [15], nanographenes [16], and polycyclic aromatic hydrocarbons $[17,18]$. These nonalternant structures often contain linked 5- and 7-membered rings. It has been recognized that their unique electronic structure makes nonalternant aromatic systems highly interesting as novel (opto)electronic semiconductor materials for organic fieldeffect transistors (OFET) and photovoltaic cells (OPVC) $[19,20]$. Nonalternant structural elements also occur in graphene in the form of 5-7 defects [21,22]. Theoretical studies have predicted that various properties of graphene depend on the presence of these nonalternant structural elements, including electron transport properties [23,24], mechanical stability [25,26], magnetism [27], and chemical reactivity [28].

While interfaces between metals and alternant organic semiconductor materials have been widely studied [29-33], up until now it has not been known how the surface chemical bond is influenced by nonalternant structural elements. To address this fundamental question, we perform a direct comparison between two isomeric aromatic molecules on a $\mathrm{Cu}(111)$ surface. One of these isomers, azulene, is a prototypical nonalternant aromatic system with a 5-7 ring structure, while naphthalene, with its 6-6 ring structure, serves as its alternant counterpart (Fig. 1). Some previous work exists for naphthalene on $\mathrm{Cu}(111)$ [34-38] but not for azulene. Copper was chosen as a model substrate because of its frequent use for the epitaxial growth of graphene [39-41] or the on-surface synthesis of carbon-based nanostructures [42].

Below, we show that the topology of the molecular $\pi$ system drastically influences its electronic interaction with a metal surface. It is therefore proposed that the incorporation

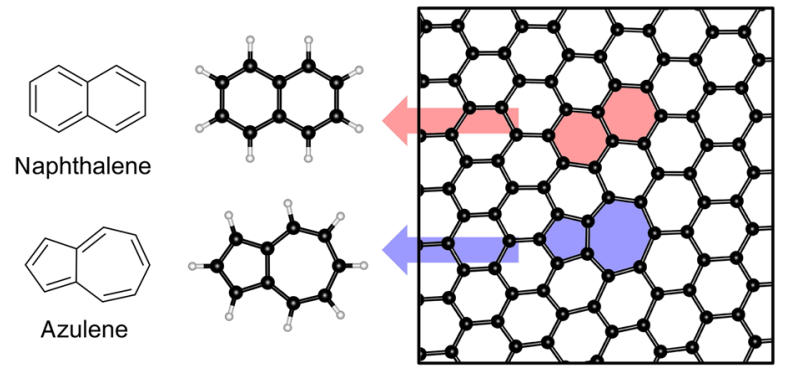

FIG. 1. Right, graphene lattice with an embedded nonalternant 5-7 defect, highlighted in blue. If this structural element is cut out of graphene and terminated with hydrogen atoms, the resulting molecule is azulene, shown on the left. Its isomer naphthalene serves as the complementary model system for the regular, alternant 6-6 structure, highlighted in red. of nonalternant structural elements in molecular semiconductors can be used to control and to optimize performancerelated properties of functional metal-organic interfaces. Recent achievements in the synthesis on novel, structurally complex, nonalternant, aromatic molecules $[16,19,20,43]$ show that this is a feasible and promising approach.

For a quantitative comparison of the adsorbate-metal bonds of aromatic 5-7 and 6-6 carbon skeletons, we determine the adsorbate-substrate bond distances with the normal incidence $\mathrm{x}$-ray standing wave (NIXSW) technique and noncontact atomic force microscopy (nc-AFM), while adsorbate-substrate bond energies are measured by temperature-programmed desorption (TPD). The electronic structures are analyzed by x-ray and UV photoelectron spectroscopy (XPS/UPS) in combination with near-edge $\mathrm{x}$-ray absorption fine-structure (NEXAFS) measurements. Complemented by dispersion-corrected density functional theory (DFT) calculations, our results provide a detailed understanding of the surface chemical bond and its dependence on the $\pi$ topology.

\section{METHODS}

\section{A. Experimental methods}

The interaction of azulene and naphthalene with $\mathrm{Cu}(111)$ was studied under ultrahigh vacuum (UHV) conditions. Azulene (Sigma-Aldrich, purity $>99.0 \%$ ) and naphthalene (Sigma-Aldrich, purity $>99.7 \%$ ) were introduced into the vacuum systems through leak valves after initial pumpfreeze-thaw cycles of the reservoirs. A polished $\mathrm{Cu}(111)$ single-crystal surface (purity $>99.9999 \%$, roughness $<0.03 \mu \mathrm{m}$, orientation accuracy $<0.1^{\circ}$, from MaTecK/ Germany) was prepared by iterated sputtering with $\mathrm{Ar}^{+}$ ions $(0.5-1 \mathrm{keV}, 5-15 \mu \mathrm{A}, 30 \mathrm{~min})$ and annealing (800$830 \mathrm{~K}, 15 \mathrm{~min}$ ). Surface cleanliness and structure were confirmed by XPS, low energy electron diffraction, and scanning tunneling microscopy (STM). Sample temperatures were measured with a type $\mathrm{K}$ thermocouple directly mounted to the $\mathrm{Cu}$ single crystal. Coverages are given in monolayers (ML). The coverage was determined by a consistent routine using XPS, nc-AFM, and TPD measurements. For a detailed description, see the Supplemental Material [44].

NIXSW measurements were performed at the undulator beam line I-09 at Diamond Light Source in Didcot, UK, using a VG Scienta EW4000 HAXPES hemispherical electron analyzer for photoelectron detection, which is mounted at $90^{\circ}$ with respect to the incident x-ray beam. Nondipolar effects were neglected in the data analysis, which was performed using the software package Torricelli [45]. The sample temperature was approximately $150 \mathrm{~K}$, which led to a Bragg energy of $2980 \mathrm{eV}$ for the $\mathrm{Cu}(111)$ lattice planes.

TPD measurements were carried out with a HIDEN EPIC 1000 mass spectrometer mounted inside a differentially pumped cryoshroud cooled to $80 \mathrm{~K}$ with $1-\mathrm{N}_{2}$. This 
setup is a variant of line-of-sight mass spectrometry [46] and ensures that molecules hitting the inner wall of the cryoshroud stick there, such that the mass spectrometer detects only molecules with a straight trajectory between sample and detector. This leads to improved quality of the TPD traces and is the basis for their quantitative analysis.

XPS was performed with monochromatic $\mathrm{Al}-\mathrm{K}_{\alpha}$ radiation using a SPECS XR $50 \mathrm{M}$ x-ray anode, a FOCUS 500 monochromator, and a PHOIBOS 150 electron energy analyzer equipped with an MCD-9 multichanneltron detector. Work functions were measured with He-I radiation from a UVS 10/35 gas discharge lamp. UPS was performed at the PM4 dipole beam line with the LowDosePES end station at the synchrotron radiation facility BESSY II (Helmholtz-Zentrum Berlin) using a Scienta ArTOF angleresolved time-of-flight spectrometer. A photon energy of $16.5 \mathrm{eV}$ was used.

NEXAFS spectroscopy was also performed at BESSY II using the HE-SGM dipole beam line, which provides linearly polarized radiation with a polarization factor of 0.91 and an energy resolution of $300 \mathrm{meV}$ at the carbon K-edge. The partial electron-yield (PEY) mode was used with a retarding field of $-150 \mathrm{~V}$ and a channeltron detector voltage of $2.2 \mathrm{keV}$. Further information on the data treatment can be found in the Supplemental Material [44].

For variable-temperature scanning tunneling microscopy (VT-STM), a SPECS 150 Aarhus STM with SPC 260 electronics was used. VT-STM measurements were performed at a sample temperature of $150 \mathrm{~K}$ using an etched tungsten tip conditioned by initial $\mathrm{Ar}^{+}$sputtering and pulsing. The reported bias voltages refer to the sample. The STM images were recorded in constant-current mode and were processed with WSxM v5.0 D8.4 [47] by carefully using flatten and plane tools, scale adjustment, and slightly filtering with a Gaussian filter.

The nc-AFM images were taken with an Omicron NanoTechnology LT-STM/AFM with a commercially available qPlus sensor, operating at approximately $4.6 \mathrm{~K}$ in UHV with an average pressure of $5 \times 10^{-10}$ mbar. The baked qPlus sensor $\left(3 \mathrm{~h}\right.$ at $\left.120^{\circ} \mathrm{C}\right)$ had a quality factor of $\mathrm{Q} \approx 30000$, a resonance frequency of $f_{0}=21922 \mathrm{~Hz}$, and a peak-to-peak oscillation amplitude of less than $1 \AA$. Tip conditioning was accomplished with controlled crashes into the copper surface and bias pulses until the STM resolution was satisfactory. The tip apex was functionalized with a CO molecule. The AFM was operated in constantheight mode, and AFM images show the frequency shift $(\Delta f)$ with respect to the resonance frequency. During the AFM scan, the tunneling current was also measured, which gives a constant-height STM scan.

\section{B. Density functional theory calculations}

Periodic density functional theory calculations of azulene and naphthalene on $\mathrm{Cu}(111)$ were performed with the Vienna Ab Initio Simulation Package (VASP) [48-51]. The
PBE functional [52] was used in combination with the third-generation van der Waals dispersion correction by Grimme (DFT-D3) [53] and the projector-augmented wave (PAW) ansatz [54,55] for the atomic cores. Further details can be found in the Supplemental Material [44].

$\mathrm{XP}$ and $\mathrm{x}$-ray absorption spectra were calculated using the pseudopotential plane-wave code CASTEP-17.1 [56]. For the XPS chemical shifts, the delta self-consistent field (DeltaSCF) method of constraining electronic occupations to resemble full core-hole excitations was used. NEXAFS simulations were performed using on-the-fly generated USPPs and the CASTEP module ELNES [57] and the transition-potential approach [58,59]. For more details on the computational settings, analysis, and implementation, see the Supplemental Material [44] and Diller et al. [60] as well as Maurer and Reuter [61]. Furthermore, the Supplemental Material [44] contains additional NEXAFS simulations obtained by a more approximate method.

\section{RESULTS}

\section{A. Adsorbate-substrate bond distances (adsorption heights)}

The vertical distance of an adsorbed molecule from the surface is an important parameter for the quantitative characterization of the adsorbate-substrate bond. To measure the adsorption heights of azulene and naphthalene over the $\mathrm{Cu}(111)$ surface [Fig. 2(a)], we used the NIXSW technique. This method utilizes the standing $\mathrm{X}$-ray wave field generated by a crystalline substrate when near-normal incidence $\mathrm{x}$ rays undergo Bragg diffraction on a set of lattice planes $\mathrm{H}=$ $(h k l)$ with a distance of $d^{\mathrm{H}}$ [62]. The standing wave field can be vertically shifted by $d^{\mathrm{H}} / 2$ by scanning the photon energy $E$ through the Bragg energy [62].

At any certain adsorption height, the photon field intensity will therefore change in a characteristic way during such a scan. Hence, one expects characteristic x-ray absorption profiles for different adsorption heights since the atomic absorption is proportional to the local photon intensity. By recording the photoelectron yield of any core level of this species, which is (within the dipole approximation) proportional to the atomic absorption, one can measure the $\mathrm{x}$-ray absorption profile at the position of the adsorbate atom and obtain the height $D^{\mathrm{H}}$ of this atomic species relative to the substrate diffraction plane locations. The variation of the atomic absorption $I(E)$ can be calculated with dynamical diffraction theory and follows the equation

$$
I(E)=1+R+2 \sqrt{R} \cdot F^{\mathrm{H}} \cdot \cos \left(\Phi+2 \pi \cdot P^{\mathrm{H}}\right),
$$

where $R=R(E)$ is the reflectivity and $\Phi=\Phi(E)$ is the phase of the standing wave field $[63,64]$.

Data analysis on the basis of Eq. (1) provides the coherent position $P^{\mathrm{H}}$ and the coherent fraction $F^{\mathrm{H}}$. The coherent position $P^{\mathrm{H}}$ equals $D^{\mathrm{H}}$ modulo $d^{\mathrm{H}}$, i.e., $D^{\mathrm{H}} / d^{\mathrm{H}}=$ $n+P^{\mathrm{H}}(n=0,1,2 \ldots)$ In the case of single-site adsorption, 

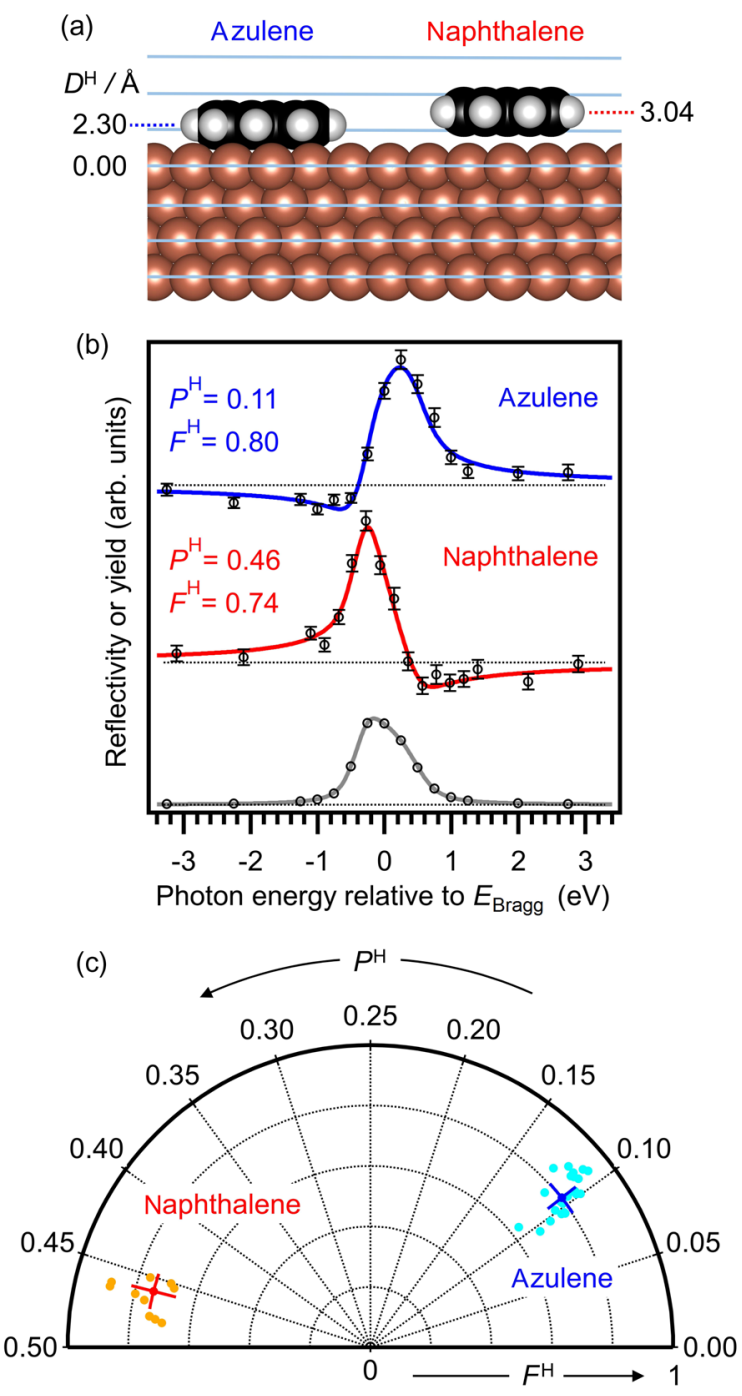

FIG. 2. Adsorption heights from NIXSW measurements for azulene and naphthalene on $\mathrm{Cu}(111)$, coverage 1 ML. (a) Spacefilling adsorbate models with the heights true to scale as obtained from NIXSW. (b) C 1s photoelectron yields for azulene (top) and naphthalene (center) along with the x-ray reflectivity $R(E)$ (bottom) as a function of the photon energy around the Bragg energy $E_{\mathrm{Bragg}}$. The yield data are shown as open circles (with error bars); the solid blue and red lines are fits with Eq. (1). (c) Argand diagram with the results of the individual measurements in light colors and the averaged value in bold colors, with error bars.

i.e., when each atomic species occupies only one adsorption height (this is the relevant case for the systems discussed here), $F^{\mathrm{H}}$ is a measure for the scattering of the real heights around the coherent position. A coherent fraction of $F^{\mathrm{H}}=1$ means that all contributing atoms have exactly the same height $D^{\mathrm{H}}$, whereas a fraction of $F^{\mathrm{H}}=0$ means that they have a random height distribution. Note that the latter is not necessarily true if (at least) two clearly different adsorption heights occur. In this case, averaging of coherent positions and fractions has to be performed in an Argand vector diagram [62-65].
For our systems, we used the background corrected total intensity of the $\mathrm{C} 1 \mathrm{~s}$ spectral region to obtain the photoelectron yield curve for each individual NIXSW scan. A typical yield curve is shown in Fig. 2(b). A distinction between the different carbon atoms within one molecule was not possible because the core level shifts were too small compared to the available energy resolution. Note that we have not performed any correction for nondipolar parameters in our analysis since the NIXSW data were recorded close to grazing emission geometry $\left(90^{\circ}\right.$ between incident $\mathrm{x}$-ray beam and the analyzer). In this geometry, nondipolar effects are minimal.

The results of the analysis are summarized in the Argand diagram in Fig. 2(c). The coherent position for azulene at monolayer coverage is $P^{\mathrm{H}}=0.11 \pm 0.01$, which yields an adsorption height of $D^{\mathrm{H}}=2.30 \pm 0.03 \AA$. This number was derived using $d^{\mathrm{H}}=2.08 \AA$ for the $\mathrm{Cu}(111)$ surface at the measurement temperature of $150 \mathrm{~K}$. The corresponding coherent fraction of $F^{\mathrm{H}}=0.80 \pm 0.06$ is in a typical range for a rather homogeneous contribution of the adsorption heights. For the naphthalene monolayer, the analysis gives a coherent position of $P^{\mathrm{H}}=0.46 \pm 0.01$, from which an adsorption height of $3.04 \pm 0.03 \AA$ is obtained. The coherent fraction is similar, with $F^{\mathrm{H}}=0.74 \pm 0.08$.

Comparison of these values [see also Fig. 2(a)] reveals a much lower adsorption height for azulene. A shorter adsorbate-substrate bond distance typically indicates a stronger bond [66], which agrees with the higher desorption energy of azulene as discussed below. The adsorption height for naphthalene is quite similar to the sum of the van der Waals radii of a carbon and a copper atom $(3.10 \AA)$ [67]. In contrast, the adsorption height of azulene, $2.30 \AA$, is closer to known organometallic carbon-copper bond lengths of approximately $2.10 \AA$ [67]. All these considerations lead to the conclusion that naphthalene engages only in dispersive interaction with the $\mathrm{Cu}(111)$ surface, whereas azulene forms a chemical bond.

NIXSW as a laterally integrating technique averages over a large number of molecules. Later, we present nc-AFM data that qualitatively confirm the height difference between azulene and naphthalene on the single-molecule level.

\section{B. Adsorbate-substrate bond energies}

Another quantitative parameter describing the strength of the adsorbate-substrate interactions is the activation energy for desorption, which can be derived from temperatureprogrammed desorption (TPD) data [68-70]. At low submonolayer coverages, the TPD traces of both molecules are governed by first-order desorption kinetics [Figs. 3(a) and $3(\mathrm{~b})]$. As can be seen, the low-coverage desorption maxima occur at $520 \mathrm{~K}$ for azulene and at $340 \mathrm{~K}$ for naphthalene. Qualitatively, this large temperature difference shows that the adsorbate-substrate interaction is substantially higher for the 5-7 isomer. 

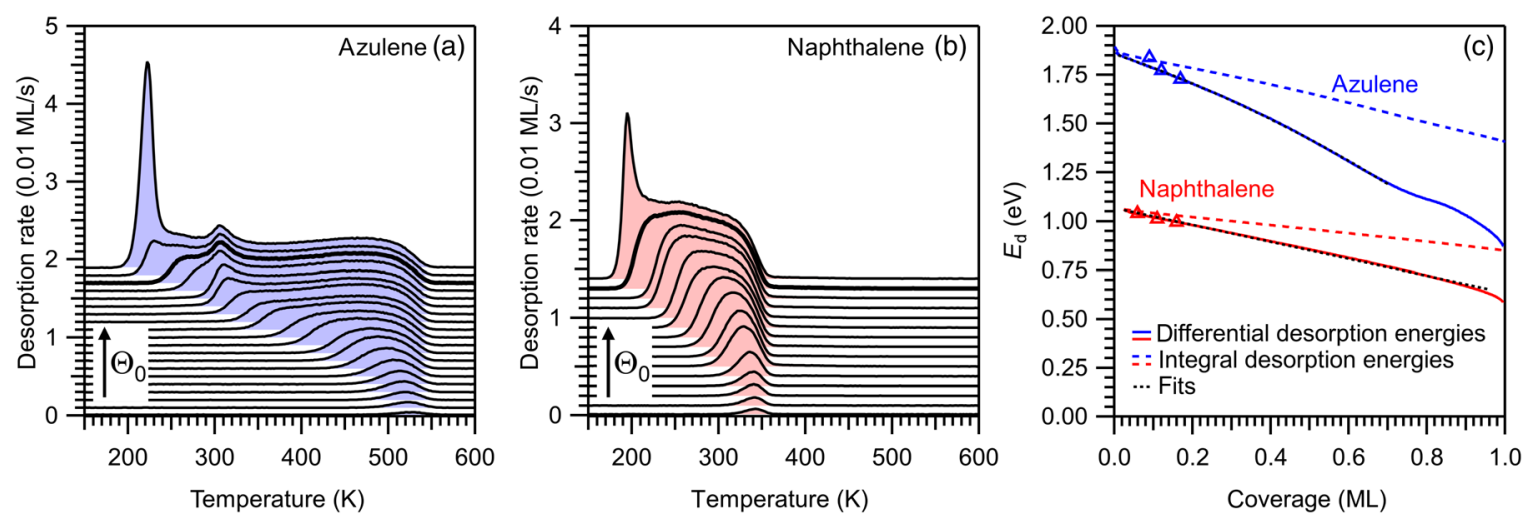

FIG. 3. TPD traces of (a) azulene and (b) naphthalene on $\mathrm{Cu}(111)$, with a heating rate $1.0 \mathrm{~K} / \mathrm{s}$. Curves with different initial coverage $\Theta_{0}$ are shifted along the vertical axis for clarity, but we start with a zero desorption rate in each case. The monolayer coverages are marked by bold lines. The other initial coverages are listed in Tables S II in the Supplemental Material [44]. (c) Desorption activation energies as a function of coverage for azulene (blue) and naphthalene (red). Solid lines: Differential desorption energies. Dashed lines: Integral energies for comparison with DFT calculations. Black dotted lines: Fits of the differential desorption energies with the equations mentioned in the text. Triangles: Energies from HRV analysis. Note that HRV is only possible at low coverages, where the peaks have a regular first-order shape.

With increasing coverage, the TPD traces of both molecules broaden towards lower temperatures. The effect, which is attributed to lateral intermolecular repulsion, is more pronounced for azulene. Its monolayer TPD trace reaches a width of $330 \mathrm{~K}$, compared to $180 \mathrm{~K}$ for naphthalene (bold lines). Lateral intermolecular repulsion in adsorbates on metal surfaces is usually dominated by a dipole-dipole interaction between vertical dipoles created by electron transfer between molecules and surface, as well as by the pillow (or pushback) effect. This effect is caused by Pauli repulsion between the electrons in the molecule and those in the metal [71-74]. Apparently, these effects play a larger role for azulene than for naphthalene. The vertical dipole moments are also related to the adsorbate-induced workfunction change. We show below that azulene causes a larger work-function change than naphthalene, in line with the stronger lateral repulsion of the former.

The high-coverage TPD traces of azulene show an additional desorption maximum at $300 \mathrm{~K}$, which is attributed to a compressed phase occurring close to monolayer saturation (above 0.7 ML). This phase can also be observed in the nc-AFM images discussed below. Above monolayer coverage, narrow second-layer peaks occur at $220 \mathrm{~K}$ (azulene) and $190 \mathrm{~K}$ (naphthalene).

Quantitative analysis of the TPD spectra gives access to the desorption activation energy $E_{\mathrm{d}}$ as a measure of the adsorbate-substrate bond energy [68-70]. The most rigorous analyses, the so-called complete methods $[75,76]$, are not suitable here because of the strong lateral repulsion [70]. Instead, we use an alternative approach, which provides the coverage-dependent desorption energy from a single TPD trace $[70,77]$. The resulting curves for azulene and naphthalene are shown in Fig. 3(c) (solid lines). The desorption energies in the zero-coverage limit, $E_{\mathrm{d}}^{0}$, are $1.86 \mathrm{eV}$ for azulene and $1.07 \mathrm{eV}$ for naphthalene. This confirms that azulene forms a much stronger bond to the metal surface than naphthalene. While both curves show a substantial decrease of $E_{\mathrm{d}}$ with increasing coverage as a result of the lateral repulsion, the effect is more pronounced for azulene. In the case of naphthalene, $E_{\mathrm{d}}$ decreases nearly linearly with coverage following the equation $E_{\mathrm{d}}=(1.07-0.44 \Theta) \mathrm{eV}$, where $\Theta$ is in units of monolayers. In contrast, the desorption energy of azulene is well described by the second-order polynomial $E_{\mathrm{d}}=\left(1.86-0.67 \Theta-0.40 \Theta^{2}\right) \mathrm{eV}$ for coverages up to $0.75 \mathrm{ML}$. Figure 3(c) also shows the integral desorption energies necessary for comparison with DFT results (dashed lines).

The desorption prefactors used for the analysis are determined by heating rate variation (HRV) analysis [68], which is only meaningful for the regular first-order peaks in the low-coverage range. The related data are presented in the Supplemental Material [44]. For the respective coverages, the HRV analysis also provides the desorption energies [triangles in Fig. 3(c)], which agree well with the other data.

\section{Occupied electronic states: Photoelectron spectroscopy}

The very different adsorption energies and heights of azulene and naphthalene are expected to correspond to characteristic differences in the electronic structure. First, we focus on the molecules in a thick multilayer, i.e., without the influence of the metal surface. The multilayer C 1s XP spectra of azulene and naphthalene are compared in Fig. 4(a). The $\mathrm{C} 1 \mathrm{~s}$ signal of azulene has a broader shape with a distinct shoulder at the low binding energy side. This shape can be understood by theoretical modeling. For this aim, we calculate the relative peak positions for the different carbon atoms in the molecule by DFT. The details of the calculation and the theoretical modeling can be found in the Supplemental Material [44]. The results are in agreement with previously reported values [78]. While these calculations are performed for the isolated molecules, this 

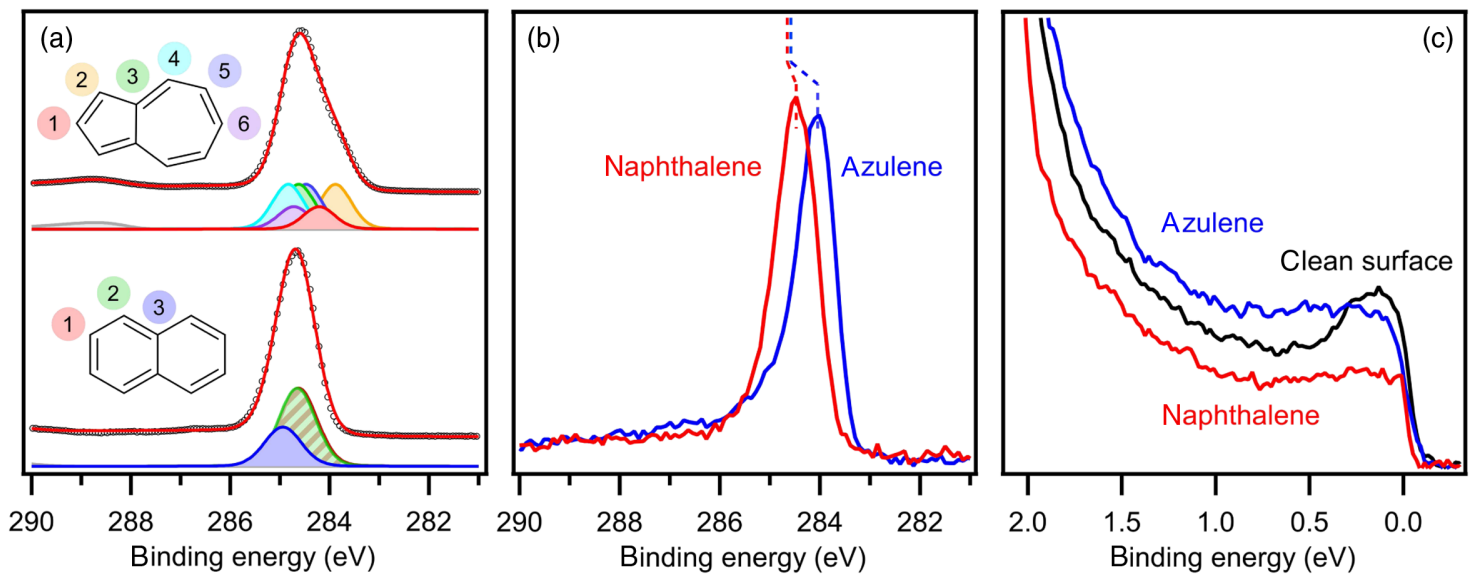

FIG. 4. Photoelectron spectra: (a) Multilayer C 1s XP spectra of azulene and naphthalene, compared with a theoretical model based on DFT calculations. Black circles are experimental data; red lines are fitted results from DFT-based model calculations. The colors of the component peaks correspond to the colors of the labels on the molecular formulas. The shake-up satellite of azulene is shown in grey. (b) $\mathrm{C} 1 \mathrm{~s} \mathrm{XP}$ spectra of monolayers of azulene and naphthalene on $\mathrm{Cu}(111)$. The dotted lines indicate the shifts relative to the multilayer peak positions. (c) UP spectra of azulene and naphthalene monolayers and of the clean $\mathrm{Cu}(111)$ surface, taken with a photon energy of $16.5 \mathrm{eV}$.

simplification appears to be justified considering that the intermolecular interactions in the multilayer are relatively weak and thus have only little influence on the electronic energies and transitions. The resulting theoretical model agrees well with the experimental data for azulene. It further reveals that the shoulder at low binding energies is associated with the five-membered ring. For naphthalene, the calculations of the $\mathrm{C} 1 \mathrm{~s}$ spectrum are performed in the same way and confirm the narrower peak shape of the experimental spectrum. In the azulene multilayer spectrum [Fig. 4(a)], a shake-up satellite at $289 \mathrm{eV}$ is shown in grey. The corresponding satellite for naphthalene appears above $290 \mathrm{eV}$ and is thus not visible in this plot. However, it can be seen in an extended plot in Fig. S3 of the Supplemental Material [44].

In the monolayer, the differences between the XP spectra of azulene and naphthalene are even more pronounced. As can be seen in Fig. 4(b), the naphthalene signal is shifted by only $-0.2 \mathrm{eV}$ relative to its multilayer position and is only slightly asymmetric. In contrast, the azulene peak shifts by $-0.5 \mathrm{eV}$ and develops a strong asymmetry. The asymmetric peak shape is attributed to the interaction of the $\mathrm{C} 1 \mathrm{~s}$ core hole with electron density close to the Fermi edge [79]. This is an indication for a distinctly different electronic valence structure of azulene compared to naphthalene when adsorbed on $\mathrm{Cu}(111)$. In an initial-state picture, the shift of the $C$ 1s signal of azulene towards lower binding energies suggests a transfer of negative charge from the surface to the molecule. Further evidence for a negative charging of the molecule will be presented below. In addition, the closer distance of the azulene molecule to the surface will likely cause increased final-state screening, which would also lead to a shift to lower binding energy.

Synchrotron-based valence photoelectron spectroscopy (PES) was used for the direct probing of the occupied valence electronic structure. In the PE spectra, the molecular states already occupied in the free molecules cannot be identified because they have binding energies above $2 \mathrm{eV}$ and thus are obscured by the d-band of the substrate. Nevertheless, the important changes in the valence electronic structure close to the Fermi edge are quite visible [Fig. 4(c)]. For azulene/ $\mathrm{Cu}(111)$, the entire range between the $\mathrm{Cu}$ d-band and the Fermi edge experiences a massive rise in intensity with a broad maximum around $0.3-0.4 \mathrm{eV}$. In contrast, naphthalene only causes attenuation of the region between 0 and $2 \mathrm{eV}$, including the surface state. The occurrence of broad, adsorbate-related features for azulene, but not for naphthalene, is consistent with the calculated densities of states, as will be discussed later.

Adsorbate-induced work-function changes were extracted from He-I UPS data and show that both azulene and naphthalene lower the work function of the $\mathrm{Cu}(111)$ surface. The change is larger for azulene with $-1.07 \mathrm{eV}$ than for naphthalene with $-0.73 \mathrm{eV}$, both at full monolayer coverage. Apparently, azulene causes a larger vertical surface dipole upon adsorption than naphthalene, in agreement with the stronger intermolecular repulsion of azulene observed in TPD. The negative sign of the work-function change indicates that the pillow effect is dominant and overcompensates for any contributions by the charge transfer from the surface to the molecule.

\section{Unoccupied electronic states: NEXAFS}

Information about the unoccupied valence electronic structure of the adsorbed molecules is obtained from carbon K-edge NEXAFS spectra. Multilayers of both molecules show a distinct set of $\pi^{*}$ and $\sigma^{*}$ resonances, which are well reproduced by the theoretical calculations, as shown in Fig. 5. 

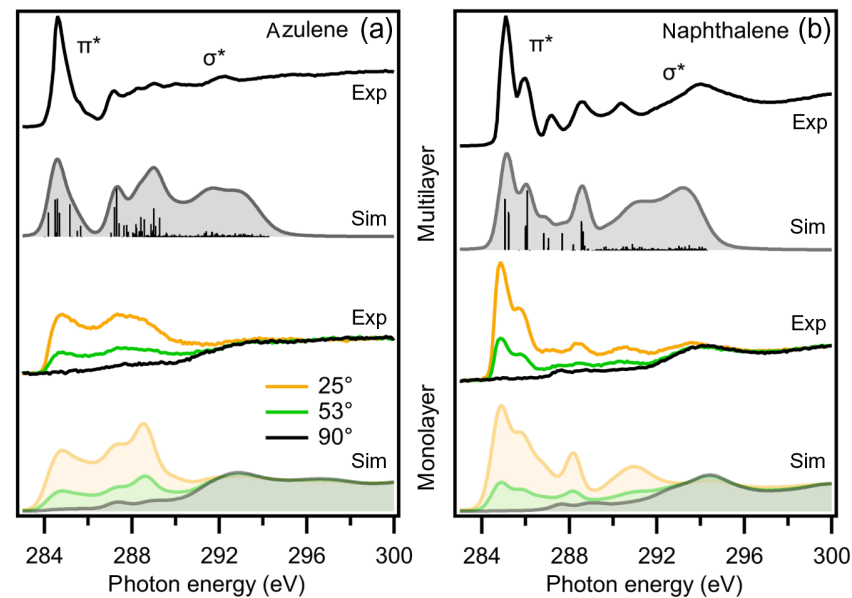

FIG. 5. Experimental carbon K-edge NEXAFS spectra and corresponding DFT simulations for (a) azulene and (b) naphthalene. Upper part: Multilayer spectra and simulations for the free molecule. The multilayer spectra were taken with the electric field vector oriented $90^{\circ}$ relative to the surface normal. Lower part: Monolayer spectra and simulations for different angles of the electric field vector relative to the surface normal as indicated. The simulations for the free molecules are displayed both as isolated excitations and after broadening; the simulations for the monolayers are only shown in broadened form. For the broadening, each excitation is represented by a pseudo-Voigt peak with an increasing width and Lorentzian contribution at higher photon energies. The simulated spectra were shifted by $-6.1 \mathrm{eV}$ to match the experimental data.

In the monolayer spectra, the $\pi^{*}$ resonances disappear when the electric field vector of the incident $\mathrm{x}$ rays is parallel to the surface (i.e., $90^{\circ}$ relative to the surface normal). This shows that the molecular planes are parallel to the surface for both azulene and naphthalene.

In the case of naphthalene, the monolayer spectrum taken with the electric field vector oriented $25^{\circ}$ relative to the surface normal closely resembles the multilayer spectrum; only a slight signal broadening is visible. This result confirms that the unoccupied valence electronic states of naphthalene are only weakly influenced by the metal surface.

For azulene, however, the shape of the $\pi^{*}$ resonance is very different in the monolayer and multilayer spectra. The narrow $\pi^{*}$ peak in the multilayer spectrum, resulting from the excitation into the LUMO and LUMO +1 of the azulene molecule, turns into a very broad monolayer feature, which is lower in intensity and covers a broad range of $5 \mathrm{eV}$. For both systems, the NEXAFS spectra are correctly reproduced by DFT-based transition potential simulations, which are discussed in detail later. A simpler approach for simulating NEXAFS spectra based only on ground-state properties can be found in the Supplemental Material [44].

\section{E. Geometric structure: Scanning probe microscopies}

Local information about the bonding situation of azulene as the model 5-7 structural element is obtained by scanning probe microscopies. These methods also provide valuable input for the DFT calculations discussed further below because there is no information in the literature about the adsorbate structure of azulene on $\mathrm{Cu}(111)$, in stark contrast to naphthalene [34-36,38,80]. In the low submonolayer range, azulene avoids the formation of ordered islands, in line with the strong lateral repulsion seen in TPD. Corresponding submonolayer STM and nc-AFM images are shown in Fig. S4 of the Supplemental Material [44]. When the coverage is increased, azulene eventually forms a longrange-ordered, commensurate $(2 \sqrt{3} \times 2 \sqrt{3})$-R $30^{\circ}$ superstructure [Fig. 6(a)]. This structure does not represent the saturated monolayer but has a coverage of only 0.65 ML. (Note that $1 \mathrm{ML}$ is defined here as the coverage in the saturated monolayer as determined by TPD.) Upon deposition of additional molecules onto this commensurate structure, the molecules are even more closely packed but lose the long-range order. This case is illustrated in the nc-AFM image in Fig. 6(b), which still shows one unit cell of the commensurate $(2 \sqrt{3} \times 2 \sqrt{3})$-R $30^{\circ}$ structure in the image center. The molecules surrounding this unit cell, however, are more densely packed and break the translational symmetry.

Formation of a commensurate phase, which is followed by a more densely packed, incommensurate phase without long-range order at full monolayer coverage, has previously been found for other organic molecules with lateral intermolecular repulsion and preference of a certain adsorption
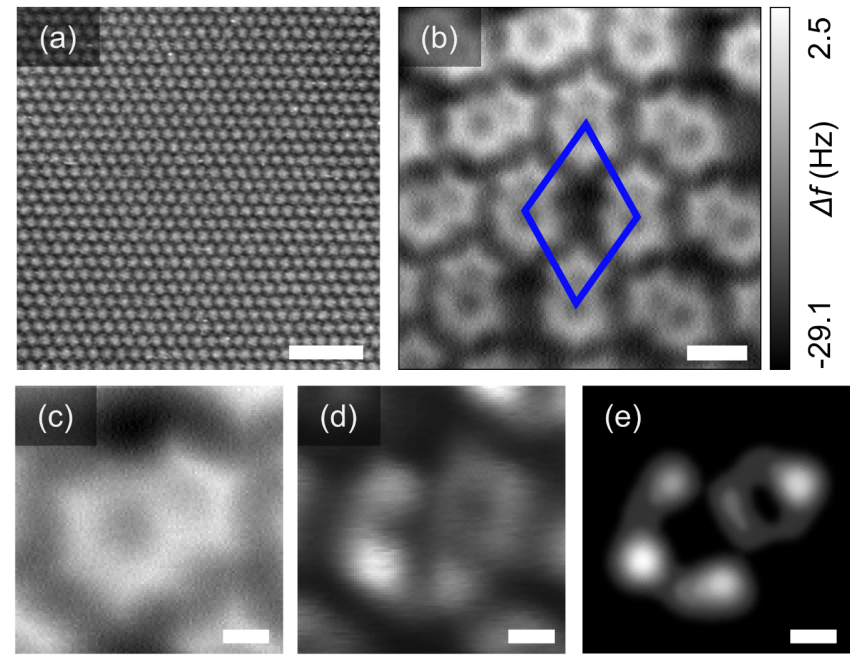

FIG. 6. STM and nc-AFM images of azulene on $\mathrm{Cu}(111)$. (a) Large-scale STM image of azulene on $\mathrm{Cu}(111)$, commensurate $(2 \sqrt{3} \times 2 \sqrt{3})-\mathrm{R} 30^{\circ}$ structure $(0.65 \mathrm{ML}), I_{\mathrm{t}}=-0.06 \mathrm{nA}$ $U_{\mathrm{t}}=-1.28 \mathrm{~V}$, scale bar $=5 \mathrm{~nm}$. (b) nc-AFM image of azulene on $\mathrm{Cu}(111)$, partially compressed phase (total coverage $0.78 \mathrm{ML})$, with an overlaid unit cell of the $(2 \sqrt{3} \times 2 \sqrt{3})-\mathrm{R} 30^{\circ}$ structure, scale bar $=0.5 \mathrm{~nm}$. Note the disordered structure with higher density around the central unit cell of the commensurate structure. (c) nc-AFM image of an azulene molecule, (d) corresponding STM constant-height image, (e) DFT Tersoff-Hamann simulation of azulene adsorbed on $\mathrm{Cu}(111)$ within an energy range of 0 to $0.1 \mathrm{~V}$ below $E_{\mathrm{F}}$. Scale bar for $(\mathrm{c}-\mathrm{e})=0.1 \mathrm{~nm}$. 
site, such as porphine on $\operatorname{Ag}(111)$ [81]. The transition from the compressed quasidisordered phase to the commensurate $(2 \sqrt{3} \times 2 \sqrt{3})$-R $30^{\circ}$ phase during desorption is associated with the small maximum around $300 \mathrm{~K}$ in the TPD curve [Fig. 3(a)].

In the nc-AFM images, the two rings of the azulene molecule can clearly be distinguished, and thus its azimuthal orientation on the surface can be determined. In the $(2 \sqrt{3} \times 2 \sqrt{3})-\mathrm{R} 30^{\circ}$ unit cell, all molecules point along the $[1 \overline{1} 0]$ direction (and symmetry equivalent directions) of the substrate.

During the nc-AFM scans, naphthalene showed a higher tendency for tip-induced lateral displacements, whereas azulene was more resistant to accidental manipulation and only occasionally showed rotation by a $60^{\circ}$ angle. Example images for both phenomena are shown in Figs. S5 and S6 of the Supplemental Material [44]. The reduced susceptibility of azulene for tip-induced displacement probably also means that it has a higher barrier for spontaneous diffusion.

In Figs. 6(c)-6(e), we compare an nc-AFM image of an adsorbed azulene molecule with the corresponding constant-height STM scan and with a DFT Tersoff-Hamann simulation [82]. The nc-AFM image in Fig. 6(c) shows the molecular structure with the five- and seven-membered rings. The STM current map in Fig. 6(d) was taken during the nc-AFM scan with negative sample bias (i.e., with electrons flowing from the sample to the tip) and shows well-defined features with lobes and nodes. Considering the bias voltage, these features must be attributed to an occupied state of the adsorbed azulene. They are well reproduced by a Tersoff-Hamann simulation using the DFT density of states (DOS) in the energy range from 0 to $0.1 \mathrm{eV}$ below the Fermi energy [Fig. 6(e)]. The shape of this occupied state closely resembles that of the LUMO of the free molecule, as will be discussed in more detail below.

The difference in adsorption height between azulene and naphthalene as found by NIXSW was also measured on the single-molecule level by comparing the positions of the minima of frequency shift distance, i.e., $\Delta f(z)$ curves [83]. The inset of Fig. 7(a) shows a STM image of codeposited azulene and naphthalene molecules. Naphthalene appears as a single bright white feature, whereas azulene is imaged as a two-segmented grey shape. The vertical and lateral position at which the feedback loop was interrupted is the same for both molecules (above the metal, the STM set point of $V=100 \mathrm{mV}$ and $I=10 \mathrm{pA}$ corresponds to $z=0 \AA$ ). The minimum $z_{\min }$ in the $\Delta f(z)$ curve for azulene occurs $0.92 \pm 0.08 \AA$ closer to the substrate than for naphthalene. This difference is highly reproducible for different tip terminations and molecules. Considering the margins of error, this value is only slightly larger than the difference in the NIXSW heights of $0.74 \pm 0.06 \AA$. The deviation between NIXSW and AFM values is possibly a temperature-related effect or due to small influences of the AFM tip.
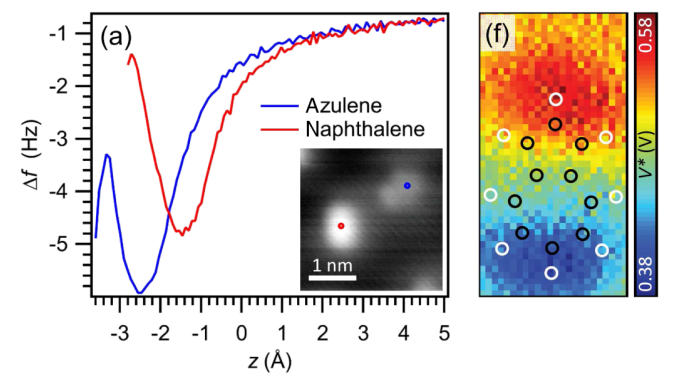

(g)
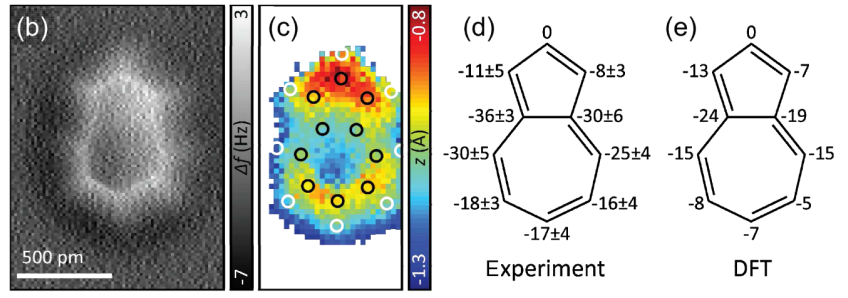

FIG. 7. (a) $\Delta f(z)$ spectra over azulene and naphthalene molecules illustrating the difference in $z_{\min }$, which is indicative of the difference in adsorption height. The inset is an STM image, where the blue and red dots indicate the positions at which the $\Delta f(z)$ spectra were taken. The STM image was acquired with $I_{\mathrm{t}}=10 \mathrm{pA}$ at $U_{\mathrm{t}}=100 \mathrm{mV}$. (b) Constant-height nc-AFM image of a single azulene molecule at $-180 \mathrm{pm}$ w.r.t. an STM set-point of $10 \mathrm{pA}$ at $100 \mathrm{mV}$. (c) $z_{\min }$ map of azulene, overlaid with carbon (black) and hydrogen (white) atom positions. (d) Experimentally obtained $z_{\min }$ height of all $\mathrm{C}$ atoms with respect to the $\mathrm{C}$ atom at the apex of the 5-membered ring, in $\mathrm{pm}$. (e) $z_{\min }$ heights of all $\mathrm{C}$ atoms with respect to the nose-C atom calculated by DFT, in pm. (f) KPFM map of a single azulene molecule, overlaid with carbon (black) and hydrogen (white) atom positions. (g) Model of the azulene molecule indicating the direction of the dipole moment.

The AFM-based height measurements also reveal a substantial adsorption-induced deformation of the azulene molecule. Figure 7(b) shows a constant-height nc-AFM image of azulene, in which the 5-7-ring structure is clearly resolved. The corresponding Fig. 7(c) shows a map with the lateral variation of $z_{\min }$, indicating that the apices of the 5- and 7-membered rings are located farther away from the surface than the $\mathrm{C}-\mathrm{C}$ bond that joins the two rings. This $\mathrm{V}$-shaped adsorption geometry is later confirmed by DFT calculations [Figs. 7(e) and 8]. A detailed comparison of the relative heights as extracted from the AFM experiments and DFT calculations is given in Figs. 7(d) and 7(e). The value for the atom with the highest position is set to zero. Both in the experiment and in theory, one long side of the molecule is located somewhat closer to the surface than the other. These results demonstrate that AFM can be used to detect vertical relaxations in single molecules in the range of a few pm.

The free azulene molecule has a considerable in-plane dipole moment of $0.8 \mathrm{D}$ [67]. To establish whether azulene adsorbed on $\mathrm{Cu}(111)$ still has an in-plane dipole moment, we performed Kelvin probe force microscopy (KPFM) experiments [83-85]. The maps extracted from these experiments show how the local contact potential 

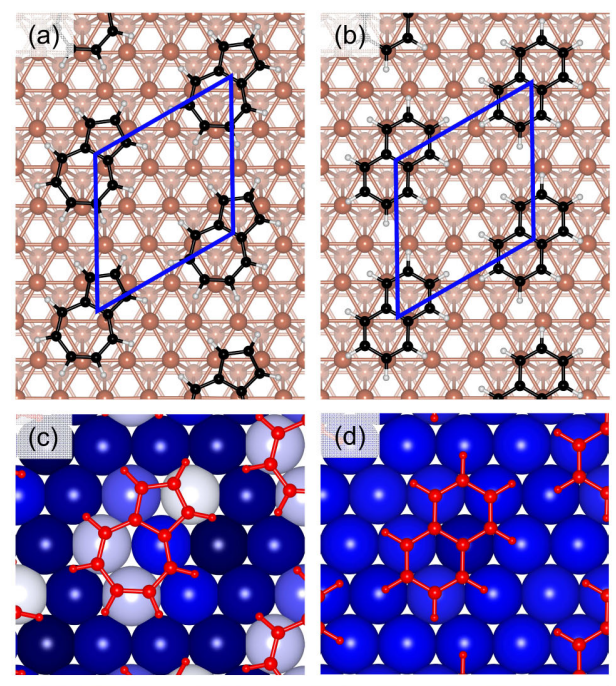

$+8.2 \mathrm{pm}$

$-6.1 \mathrm{pm}$ (e)

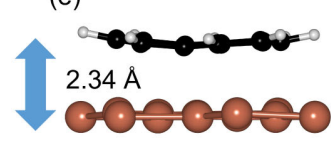

(g)

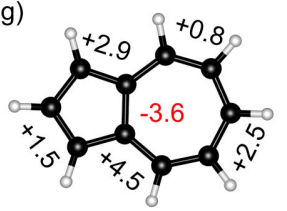

(f)

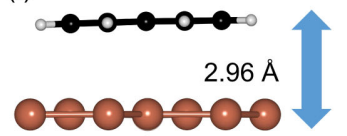

(h)

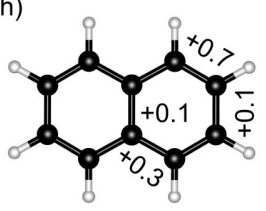

FIG. 8. Structural data for azulene and naphthalene on $\mathrm{Cu}(111)$ from dispersion-corrected DFT-D3 calculations. (a,b) Top view of the optimized $(2 \sqrt{3} \times 2 \sqrt{3})$-R30 $0^{\circ}$ structure. The most favorable adsorption site for both molecules is hcp-hcp. (c,d) Vertical displacements (in pm) of the copper atoms in the topmost layer, compared to the relaxed surface without a molecule. Positive values mean a displacement towards the molecule. (e,f) Side view of the molecule in the optimized $(2 \sqrt{3} \times 2 \sqrt{3})$-R $30^{\circ}$ structure. Azulene shows a large distortion and is much closer to the surface than naphthalene (average adsorption height $2.34 \AA$ vs $2.96 \AA$ ). $(\mathrm{g}, \mathrm{h})$ Changes of the in-plane bond lengths relative to the gas phase structure (in pm).

difference varies. This quantity is related to the electrostatic potential above the sample [84]. The KPFM maps presented here are obtained from finding the maximum $\left(V^{*}\right)$ of $\Delta f(V)$ curves extracted from a set of constant-height AFM images at increasing bias [85]. Figure 7(f) presents a $V^{*}$ map of a single azulene molecule. The $V^{*}$ for the 5membered ring is about $+0.52 \mathrm{~V}$, while for the 7-membered ring, it is $+0.42 \mathrm{~V}$. The value of $V^{*}$ represents the voltage needed to minimize the electrostatic interaction between the tip and the molecule. Above the 5-membered (7-membered) ring, a larger (smaller) positive voltage is needed to compensate for the presence of the negative (positive) charge. From the $V^{*}$ data shown in Fig. 7(f), it is evident that the adsorbed molecule has an in-plane dipole along the long molecular axis, pointing from the 5- to the 7-membered ring. The magnitude of the retained dipole moment cannot be extracted from such experiments [86].

\section{F. Theoretical analysis}

For additional insight into the surface chemical bond of azulene and naphthalene, dispersion-corrected periodic density functional theory calculations (DFT-D3) [53] were performed for the ordered $(2 \sqrt{3} \times 2 \sqrt{3})-\mathrm{R} 30^{\circ}$ structure, which was found in the STM images of azulene/ $\mathrm{Cu}(111)$ [Fig. 6(a)]. This structure is not observed for naphthalene/ $\mathrm{Cu}(111)$, but as the $(2 \sqrt{3} \times 3)$-Rect structure known in the literature has the same coverage, it is also used here for better comparability [34,36]. For both molecules, the same preferential adsorption site is found. In this geometry, the molecules adsorb with each ring above an hep hollow site, as shown in Figs. 8(a) and 8(b). This result is in agreement with the nc-AFM data, which show that the molecules are aligned along a principal direction of the surface, which is also the case with this adsorption site.

Closer inspection of the adsorbate geometries reveals that the azulene molecule and the top layer of the surface undergo substantial adsorption-induced deformations. The surface copper atoms in the unit cell differ in height by up to $0.14 \AA$, as is visualized in Fig. 8(c). For comparison, naphthalene does not cause any significant deformation of the surface [Fig. 8(d)]. Azulene experiences an out-of-plane deformation, which brings the bridging carbon atoms closer to the surface than the apex atoms of the rings [Fig. 8(e)]. This was also observed with nc-AFM [Figs. 7(c) and 7(d)]. In addition, azulene shows extensive changes of the inplane bond lengths, in particular, a shortening of the bridging bond $(-3.6 \mathrm{pm})$ and elongations of the other bonds (up to $+4.5 \mathrm{pm}$ ). This can be explained by electron donation into the LUMO [as shown in Fig. 9(a)] because its bonding and antibonding contributions agree with the pattern in the bond length changes. In the case of naphthalene, the adsorption-induced deformations are much smaller [Figs. 8(d), 8(f), and 8(h)].

The adsorption height was calculated as the distance between the average height of the carbon atoms and the relaxed height of the first substrate layer without a molecule. For naphthalene, this approach gives a height of $2.96 \AA$, which is only slightly smaller than the sum of the van der Waals radii of a carbon and a copper atom $(3.10 \AA)$ and thus is consistent with the van der Waals character of the naphthalene-copper interaction. In the case of azulene, the calculated distance of $2.33 \AA$ is closer to the sum of the covalent radii of a carbon and a copper atom $(2.20 \AA)$, in line with the much stronger bond of azulene. Both calculated values are in excellent agreement with the NIXSW results of $2.30 \AA$ for azulene and $3.04 \AA$ for naphthalene.

In this comparison, we have considered that the standing $\mathrm{x}$-ray wave is formed by the bulk lattice planes, and therefore, the experimental height value is the distance 
between the carbon atoms of the molecule and the ideal, unrelaxed surface layer. For $\mathrm{Cu}(111)$, the vertical relaxation of the adsorbate-covered first layer proved to be very small in our calculations (less than $0.01 \AA$ ), and thus the relaxation correction is actually negligible.

In the electronic adsorption energies yielded by the DFTD3 calculations, azulene shows a much higher value than naphthalene, $E_{\mathrm{ads}}=-1.79 \mathrm{eV}$ and $-1.40 \mathrm{eV}$, respectively. When zero-point vibrational energy (ZPVE) corrections are taken into account, the energy for azulene increases to $E_{\text {ads }}=-1.87 \mathrm{eV}$, while there is no change for naphthalene (because its vibrational frequencies are much less influenced by the adsorption). To compare these DFT results with TPD data, we must use the integral desorption energy at the coverage of the $(2 \sqrt{3} \times 2 \sqrt{3})-\mathrm{R} 30^{\circ}$ structure used in the DFT calculations $(0.65 \mathrm{ML})$ and make the (here very reasonable) assumption that the adsorption of azulene and naphthalene has no activation barrier. The corresponding experimental energies are $1.58 \mathrm{eV}$ for azulene and $0.93 \mathrm{eV}$ for naphthalene [see Fig. 3(c), dashed lines]. In comparison, theory overestimates the adsorption energy by $0.29 \mathrm{eV}$ for azulene and by $0.47 \mathrm{eV}$ for naphthalene, which is in the range of expected deviations of current DFT methods [87]. In particular, dispersion-corrected DFT adsorption energies of molecules on metal surfaces are typically overestimated and usually show errors of this magnitude $[88,89]$.

DFT also provides additional insight into the valence electronic structure of the adsorbed molecules. For azulene $/ \mathrm{Cu}(111)$, the carbon-projected DOS in Fig. 9(e) shows a substantial density of states spread out over a large energy window around $E_{\mathrm{F}}$, with only small variations in magnitude. In contrast, naphthalene has a clear gap in the carbon-projected DOS between -1.4 and $+0.5 \mathrm{eV}$ [Fig. 9(f)]. Both findings are in agreement with the UPS data in Fig. 4(c). The reason for this different behavior is related to azulene's low-lying LUMO, which is very close to the Fermi edge of $\mathrm{Cu}(111)$, as shown in Fig. 9(e). As a result, the LUMO is pulled below $E_{\mathrm{F}}$ and is partially filled with electrons from the surface, as can be seen in the charge density difference plot [Figs. 9(a) and 9(b)]. Comparison with a related plot for naphthalene using the same isosurface value shows no visible charge transfer [see Figs. 9(c) and 9(d)]. If a much lower isosurface value is chosen, the so-called pillow effect (i.e., the pushback of electron density between the molecule and surface caused by Pauli repulsion [71]) can be seen for both molecules, but it is much larger for azulene. These additional charge density difference plots are shown in Fig. S7 of the Supplemental Material [44].

The participation of the frontier orbitals in the surface chemical bond is illustrated in Figs. 10(a) and 10(b), which show the DOS projected onto the molecular orbitals of the free molecules. Again, drastic differences can be seen between azulene and naphthalene. The frontier orbitals of azulene [Fig. 10(a)] undergo substantial energetic broadening in the adsorbed state. As a result, the LUMO and (a)

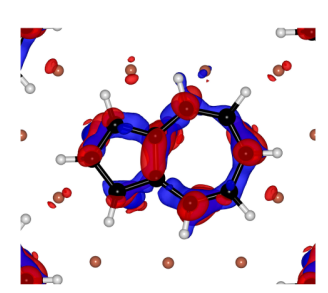

(b)
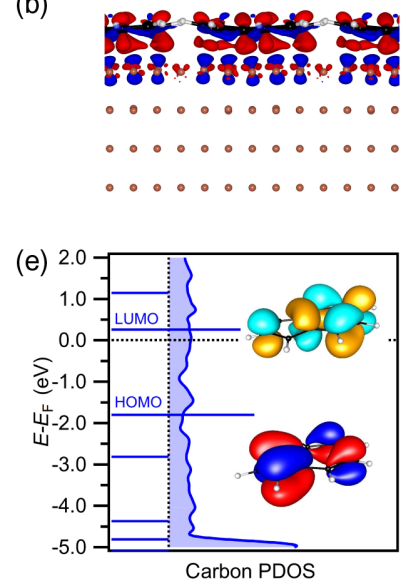

(c)

Naphthalene

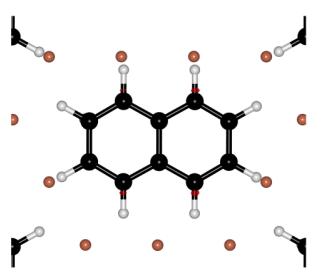

(d)

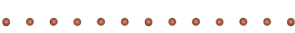

...........
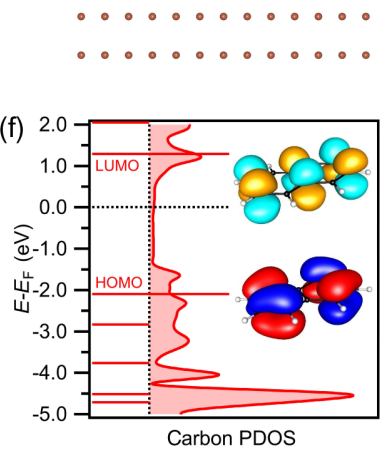

FIG. 9. Electronic structure of azulene and naphthalene on $\mathrm{Cu}(111)$ from DFT calculations. (a,b) Charge density difference plots for azulene. (c,d) Charge density difference plots for naphthalene. The isosurface value is $0.003 e^{-} / \AA$; electrons flow from blue to red. In panel (a), electron enrichment in the shape of the LUMO is clearly visible. (e,f) Carbon partial density of states of the adsorbed species. The horizontal bars represent the energies of the frontier orbitals of the free molecules. These energy levels are shifted to align the lowest valence orbitals for the free and the adsorbed molecules. The figure also shows images of the HOMO and LUMO as calculated by DFT.

LUMO +1 levels show large contributions below the Fermi energy. In the case of naphthalene, the frontier orbitals are much less broadened and the LUMO remains well above the Fermi energy.

The adsorption-induced changes in the valence electronic structure also have important consequences for the NEXAFS spectra, as can be seen in the MO-projected NEXAFS simulations [Figs. 10(c)-10(f)]. The LUMO and LUMO +1 contributions are greatly reduced for azulene, when adsorbed on $\mathrm{Cu}(111)$, because these orbitals are now partially occupied and therefore are not fully available for an excitation anymore. For naphthalene, adsorption does not result in substantial spectral changes. Note that the MO contributions do not seem to add up to the total spectrum for azulene on $\mathrm{Cu}$ (111) [Fig. 10(e)]. Because of the strong hybridization between the molecular orbitals and the surface, as well as final-state screening effects, the spectral contributions from states with ground-state molecular orbital character are much smaller than for the weakly interacting naphthalene.

The hybridization of the frontier orbitals also has consequences for the charge redistribution between the 


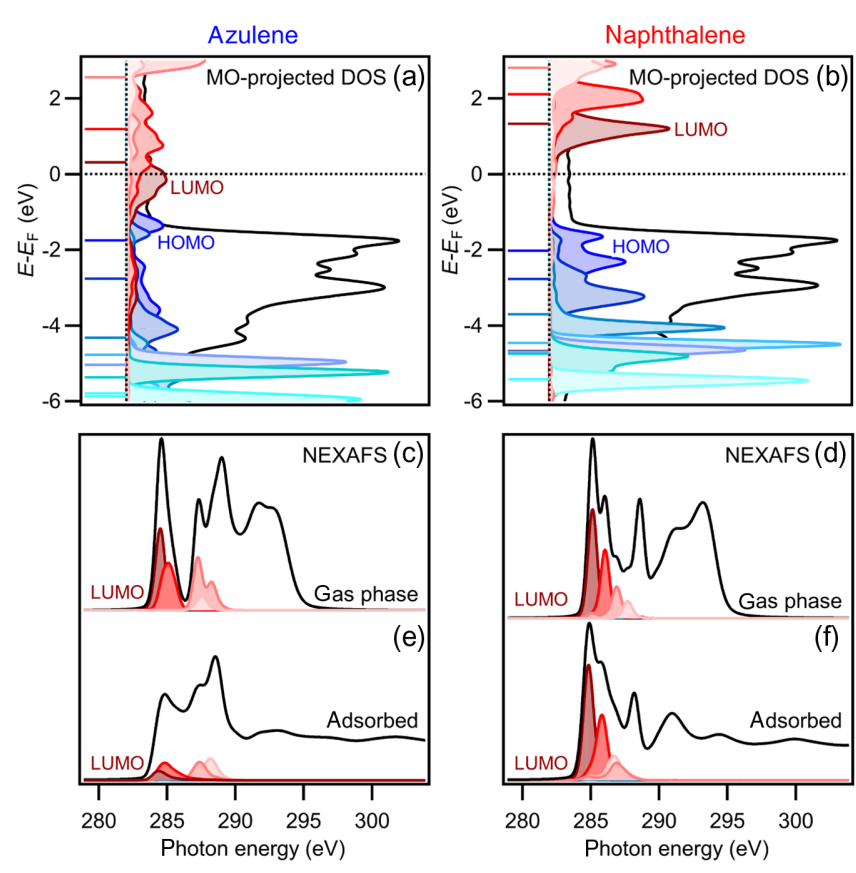

FIG. 10. MO projection analysis, left azulene, right naphthalene. Contributions of the LUMO are shown in dark red and of the HOMO in blue; higher and lower orbitals are shown in incrementally lighter colors. Total DOS and total spectrum are shown in black. (a,b) TDOS and MO-projected density of states of the adsorbed species with the energies of the molecular frontier orbitals. The nonvanishing TDOS around the Fermi energy is caused by the metal substrate. (c,d) MO-projected NEXAFS simulations of the free molecules. (e,f) MO-projected NEXAFS simulations of the adsorbed molecules. The simulated spectra are shifted by $-6.1 \mathrm{eV}$ to match the experimental data in Fig. 5 .

surface and the molecule. The charge transfer is quantified using two different methods. The Bader analysis method [90] predicts that azulene receives 0.49 negative elementary charges $\left(e^{-}\right)$from the surface, resulting in a net negative charge at the molecule. In contrast, naphthalene shows only a very small charge transfer of $0.06 e^{-}$from the molecule to the surface, i.e., in the opposite direction. Using an alternative approach, we integrate the MO projection in Figs. 10(a) and 10(b) up to $E_{\mathrm{F}}$, including the pulled-down contributions of LUMO and LUMO+1. The resulting excess charges (negative charges) on the molecules are $1.39 e^{-}$for azulene and $0.13 e^{-}$for naphthalene, respectively. Both methods thus indicate a significant surface-tomolecule charge transfer. The partial charge is not an observable property, and it is well known that different methods give different absolute values. The chemisorbed character of azulene on $\mathrm{Cu}(111)$ means that separation of molecule and surface electronic states is not straightforward, either in real or in orbital space. However, the considerable effects observed in the NEXAFS spectrum of azulene upon adsorption indicate that the magnitude of charge transfer is probably larger than predicted by the Bader analysis method.

\section{DISCUSSION}

The combined TPD, NIXSW, PES, NEXAFS, nc-AFM, and DFT results provide a consistent picture of the surface chemical bond of azulene and naphthalene on $\mathrm{Cu}(111)$. Azulene, as a prototypical 5-7 system, forms a strong chemical bond to the copper surface, whereas naphthalene as a 6-6 system is only physisorbed. This difference is related to the topology of the $\pi$-electron system. In naphthalene, the $\pi$ system has an alternant topology, which means that all carbon atoms can be divided into two disjoint sets, such that an atom of one set binds only to atoms of the other set [14]. All aromatic systems with 6-membered rings, the benzoid systems, are alternant. In contrast, the 5-7 $\pi$ system of azulene has a nonalternant topology [Fig. 11(a)].

Alternant and nonalternant $\pi$ systems show fundamental differences in the electronic structure. The CoulsonRushbrooke theorem, which states that the energy levels of the aromatic $\pi$ system are symmetrically distributed, is

(a)
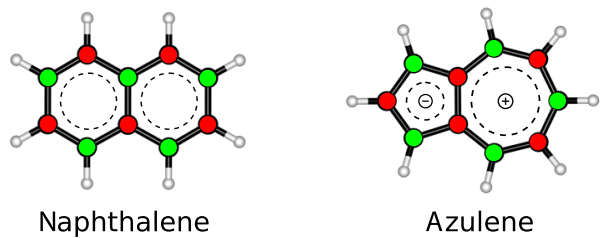

(b)

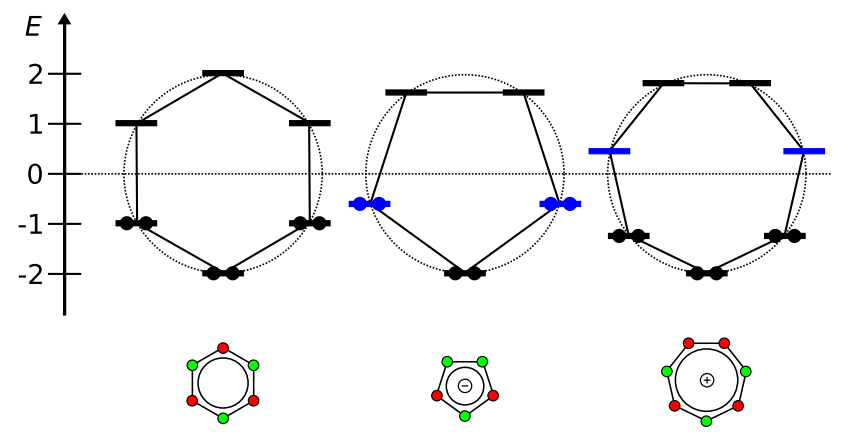

FIG. 11. (a) Alternating labeling of the carbon atoms (red, green), illustrating that the 6-6 system has an alternant and the 5-7 system has a nonalternant $\pi$ system. The dipole moment of the 5-7 system can also be understood as a consequence of the Hückel $4 n+2$ rule for aromatic systems [91], which requires transfer of one electron from the heptagon to the pentagon, such that an aromatic sextet (Clar sextet) can be formed [92]. (b) FrostMusulin diagrams [93] for 6-, 5-, and 7-membered cyclic $\pi$ systems. The HOMO level of the 5-membered ring and the LUMO level of the 7-membered ring are highlighted in blue. All $\pi$-MO energies lie on a circle, which is centered at the Coulomb integral $\alpha$ and has the radius of 2 times the Hückel exchange integral $\beta$. The energy scale is in units of $x$, with $x \equiv(\alpha-\varepsilon) / \beta$, where $\varepsilon$ is the $\pi$-MO energy. Only the 6 -membered ring is alternant and fulfills the Coulson-Rushbrooke theorem. In the 5membered system, the HOMO is lifted, and in the 7-membered system, the LUMO is lowered. The hexagon has a neutral closedshell state, whereas the pentagon and the heptagon form a closedshell anion and cation, respectively. 
violated by nonalternant species [14,94]. While alternant aromatic molecules have highly delocalized $\pi$ orbitals, these orbitals are more localized in nonalternant molecules.

The concept of alternant and nonalternant cyclic $\pi$-conjugated systems is visualized in Fig. 11(b), which compares the Frost-Musulin energy diagrams for 5-, 6-, and 7-membered cyclic $\pi$ systems [93]. As can be seen, the 6 -membered ring is alternant, and its $\pi$-orbital energies are paired, such that each occupied bonding orbital with energy $-E$ has an empty antibonding counterpart with energy $+E$. This symmetry is broken for nonalternant 5 - and 7-membered $\pi$ systems. As one of the consequences, the HOMO of the 5-membered ring is lifted, whereas the LUMO of the 7-membered ring is lowered, compared to the 6-membered, alternant system.

Qualitatively, the electronic structure of azulene can be viewed as a combination of 5- and 7-membered $\pi$ systems. From this consideration, one would expect that the HOMO-LUMO gap of azulene is considerably smaller than that of the corresponding system with two 6-membered rings, naphthalene. The increased HOMO energy should make azulene a better donor, and the reduced LUMO energy a better acceptor, than naphthalene. In addition, the frontier orbitals should be somewhat localized, with increased contributions of the 5-membered ring to the HOMO and of the 7-membered ring to the LUMO.

These anticipated properties are in agreement with the experimental observations. The localization of HOMO and LUMO in azulene causes a molecular dipole moment of $0.8 \mathrm{D}$ [67], which is a very large value for a simple hydrocarbon without heteroatoms. In contrast, naphthalene has no dipole moment. The HOMO-LUMO gap of azulene is $2.1 \mathrm{eV}$, compared to $3.5 \mathrm{eV}$ for naphthalene, as calculated by DFT.

With respect to the adsorption behavior of azulene, the energetic shift of the frontier orbitals, especially the down-shift of the LUMO, leads to the described accessibility for electron donation from the surface into the LUMO and thus to the much stronger chemical bond to the $\mathrm{Cu}(111)$ surface. The partial occupation of the LUMO also explains some of the adsorption-induced deformations, especially the striking shortening of the bridging bond [see Fig. 8(g)] because the LUMO is bonding while the HOMO is antibonding between the two shared $\mathrm{C}$ atoms [see Fig. 9(e)].

As pointed out above, the special electronic structure of azulene and the resulting strong surface chemical bond are by no means a coincidence but a consequence of the topology of its $\pi$ system, which is fundamentally different from that of naphthalene. We expect this to hold true wherever this structural element occurs, whether it be in a molecule, a graphene nanoribbon, or another $\pi$-electron system. The limitations of this molecular model system are discussed in the following for a system that is, in many ways, furthest removed from the molecules investigated: defects in an infinite graphene lattice.
The 5-7 defects embedded in the graphene lattice have the same topology as azulene, whereas the regular graphene lattice has the same topology as naphthalene (Fig. 1). Therefore, we expect the 5-7 defects in graphene to have a much stronger and more localized interaction with a metal substrate than the regular graphene. It is also possible that metal atoms deposited onto graphene bind more strongly to 5-7 defects than to regular lattice sites. In addition, electron transfer from the substrate is more likely to occur at the 5-7 defects than at defect-free areas.

Compared to a 5-7 defect in substrate-supported graphene, our molecular system, azulene, differs in two important points. First, the $\pi$ system in azulene is terminated by hydrogen atoms, and thus the area of $\pi$-electron delocalization is spatially confined. In contrast, the 5-7 defect in a graphene layer is embedded in the $\pi$ system of graphene, making the area of delocalization much larger. However, it is important to keep in mind that the effect of the topological symmetry break caused by the 5-7 defect is a spatial localization and energetic lowering of electronic states. These effects should also occur in the extended structure because the symmetry break is also present there. To verify this assumption, we performed periodic DFT calculations for a 5-7 defect embedded in a freestanding graphene layer. As can be seen in a section through the charge density, the defect interrupts the homogeneous charge distribution present in the defect-free graphene [Fig. 12(a)] and accumulates negative charge at the 5membered ring and positive charge at the 7 -membered ring [Fig. 12(b)]. The local charge accumulation on the embedded 5-7 defect, as quantified by the Hirshfeld charge analysis [95], has a similar magnitude as in the isolated 5-7 system of azulene (see the Supplemental Material [44] for details). The graphene with the embedded defect also shows additional DOS in the vicinity of the Fermi energy [Fig. 12(c)].

Another difference between the molecular nonalternant system and a 5-7 defect in an adsorbed graphene layer will be apparent in the adsorption height. While azulene can always adopt its equilibrium height, the 5-7 defect embedded in the graphene lattice (or in another extended aromatic system) will be pulled closer to the surface, but it will also be held back by the surrounding regular graphene lattice (or other alternant structure), which resides at a larger distance. This competition may further influence the electronic interaction with the substrate and related effects, such as electron transfer. Nevertheless, the molecular nonalternant system of azulene represents a highly valuable model for extended aromatic systems with embedded nonalternant structural elements because reliable quantitative information such as local interaction energies and adsorption heights are very difficult to obtain for defects in extended $\pi$-conjugated systems with the presently available experimental and theoretical methods. 

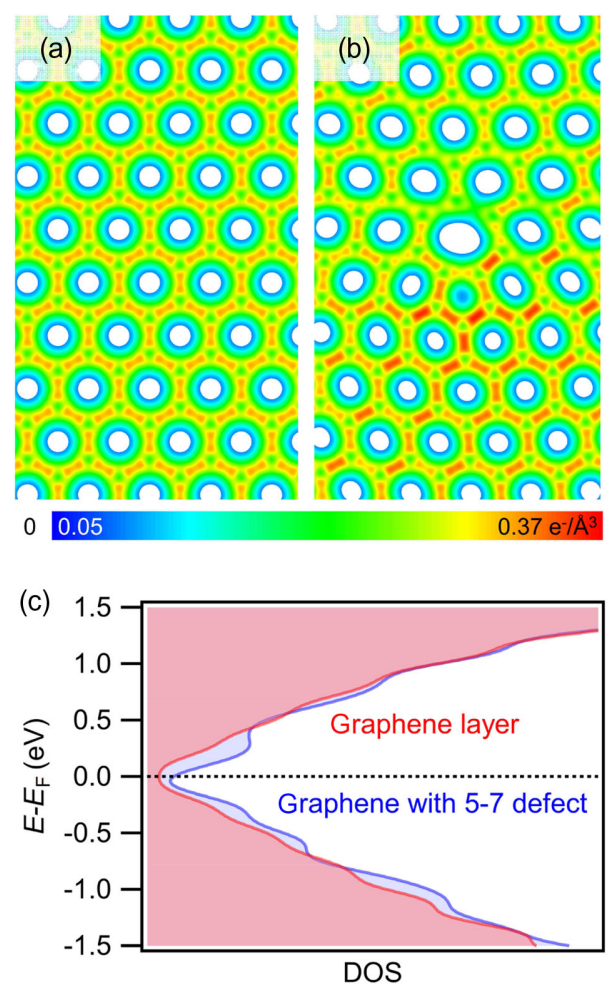

FIG. 12. Results of periodic DFT calculations for a freestanding graphene layer with and without 5-7 defects. (a) The section through the charge density for the planar ideal graphene layer and (b) the section through the charge density for the planar graphene layer with a 5-7 defect. As can be seen, the 5-7 defect accumulates negative charge (red) at the 5-membered ring and positive charge (blue/white) at the 7-membered ring. Only part of the unit cell is shown. For a complete view, see Fig. S9 of the Supplemental Material [44]. (c) Total density of states for both systems. The DOS of the defect structure shows new states around the Fermi energy. The nonzero DOS at $E_{\mathrm{F}}$ for both systems is caused by the employed electronic smearing and is a known artifact of the method [96,97].

\section{CONCLUSIONS}

Azulene as a nonalternant aromatic hydrocarbon forms a much stronger chemical bond to the $\mathrm{Cu}(111)$ surface than naphthalene as its alternant isomer. This result follows from the very different low-coverage TPD peak temperatures ( $520 \mathrm{~K}$ for azulene vs $340 \mathrm{~K}$ for naphthalene) and from the resulting zero-coverage desorption energy, which is much higher for azulene $(1.86 \mathrm{eV})$ than for naphthalene $(1.07 \mathrm{eV})$. The same trend is seen in the vertical bonding distances (adsorption heights) as measured by NIXSW and nc-AFM. The NIXSW value of $2.30 \AA$ for azulene is much smaller than the sum of the van der Waals radii and agrees with the formation of a real chemical bond. In contrast, the height of $3.04 \AA$ found for naphthalene is consistent with pure physisorption. The distances calculated by dispersioncorrected DFT agree very well with the measured heights. The calculations also show that both azulene and the surface underneath undergo substantial distortion (as is confirmed by AFM experiments), while naphthalene remains almost undistorted. Repulsive interactions between the adsorbed azulene molecules lead to a strongly coverage-dependent desorption energy, which follows the equation $E_{\mathrm{d}}=\left(1.86-0.67 \Theta-0.40 \Theta^{2}\right) \mathrm{eV}$. Naphthalene shows less repulsion, and its desorption energy is well described by the equation $E_{\mathrm{d}}=(1.07-44 \Theta) \mathrm{eV}$. Effects of the intermolecular repulsion are also visible in the submonolayer STM images. The work-function change at monolayer coverage is higher for azulene $(-1.07 \mathrm{eV})$ than for naphthalene $(-0.73 \mathrm{eV})$, indicating that azulene forms the larger surface dipole. The higher intermolecular repulsion seen for azulene is therefore likely related to increased dipole-dipole repulsion.

The differences in the surface chemical bonds of azulene and naphthalene can be rationalized on the basis of the different topologies of the molecular $\pi$ systems. Because of its violation of the Coulson-Rushbrooke theorem, azulene has a low-lying LUMO, which is close to the Fermi energy of copper. This fact leads to a strong hybridization between the LUMO and electronic states of the surface, as well as electron transfer from the surface into the LUMO. This electron transfer is confirmed by various experimental data: Probing the occupied states, the UP spectra of azulene on $\mathrm{Cu}$ (111) show an adsorption-induced, broad feature below the Fermi edge. Complementary NEXAFS studies of the unoccupied states reveal strong changes in the $\pi^{*}$ resonance, which is attenuated and broadened. Theoretical analysis shows that the new broad feature in the occupied range is related to the former LUMO and other formerly unoccupied orbitals, while the changes in the NEXAFS are due to the partial occupation of the LUMO and LUMO +1 orbitals. In addition, submolecularly resolved STM images of azulene show the shape of the former LUMO at negative bias, which confirms that this orbital is filled with electrons. This finding is also supported by STM simulations. Theoretical analysis indicates a pronounced redistribution of charge in the case of azulene and a substantial charge transfer from the surface to the molecule. In contrast, the electronic structure of naphthalene is only slightly influenced by the surface, in line with its weaker and longer surface chemical bond.

Our results show that the topology of an aromatic $\pi$ system greatly influences its interaction with a metal surface. In particular, structural elements with the nonalternant 5-7 topology can form much stronger surface chemical bonds than elements with the regular, alternant 6-6 topology. This may be true not only for aromatic molecules but also for all occurrences of nonalternant topology in carbon-based nanostructures on surfaces, in graphene nanoribbons or, in the form of 5-7 defects, in graphene itself. For all of these structures, we predict a localized surface chemical bond and local electron transfer between the substrate and adsorbate. In addition, our findings may be relevant for the optimization of the 
metal-organic interfaces that occur at electrodes in organic electronic devices. They suggest that modifying the topology of the molecular $\pi$ system represents a possible way to tune performance-related parameters such as wavefunction overlap and energy-level alignment at the electrode-semiconductor interface.

\section{ACKNOWLEDGMENTS}

Funded by the Deutsche Forschungsgemeinschaft (DFG, German Research Foundation)-Projektnummer 223848855SFB 1083. We thank the synchrotron radiation facilities BESSY-II of the Helmholtz-Zentrum für Materialien und Energie, Berlin, for allocation of beam time at the beam lines HE-SGM and PM4/LowDosePES and for financial support. The experimental support by E. Giangrisostomi and R. Ovsyannikov is gratefully acknowledged. Furthermore, we thank Diamond Light Source for access to beam line I09 (Proposal No. SI16259), and the I09 beam-line staff (T.-L. Lee, P. K. Thakur, and D. McCue) for their support during the experiment. We acknowledge computational resources from HLR Stuttgart, CSC-LOEWE Frankfurt, and HRZ Marburg.

[1] J.E. Anthony, The Larger Acenes: Versatile Organic Semiconductors, Angew. Chem. Int. Ed. 47, 452 (2008).

[2] A. Mishra and P. Bäuerle, Small Molecule Organic Semiconductors on the Move: Promises for Future Solar Energy Technology, Angew. Chem. 51, 2020 (2012).

[3] J. H. Burroughes, D. D. C. Bradley, A. R. Brown, R. N. Marks, K. Mackay, R. H. Friend, P. L. Burns, and A. B. Holmes, Light-Emitting Diodes Based on Conjugated Polymers, Nature (London) 347, 539 (1990).

[4] A. Pron and P. Rannou, Processible Conjugated Polymers: From Organic Semiconductors to Organic Metals and Superconductors, Prog. Polym. Sci. 27, 135 (2002).

[5] Z. Chen, Y.-M. Lin, M. J. Rooks, and P. Avouris, Graphene Nano-ribbon Electronics, Physica E 40, 228 (2007).

[6] B. H. Nguyen and V.H. Nguyen, Promising Applications of Graphene and Graphene-based Nanostructures, Adv. Nat. Sci. Nanosci. Nanotechnol. 7, 023002 (2016).

[7] Concepts in Molecular and Organic Electronics, MRS Proceedings, edited by N. Koch, E. Zojer, S.-W. Hla, and X. Zhu (Cambridge University Press, Cambridge, England, 2014).

[8] Organic Electronics: Materials, Manufacturing, and Applications, edited by H. Klauk (Wiley-VCH, Weinheim, 2006).

[9] Organic Electronics II: More Materials and Applications, edited by H. Klauk (Wiley-VCH, Weinheim, 2012).

[10] J. M. Gottfried, Quantitative Model Studies for Interfaces in Organic Electronic Devices, New J. Phys. 18, 111002 (2016).

[11] A. Kahn, N. Koch, and W. Gao, Electronic Structure and Electrical Properties of Interfaces between Metals and $\pi$-conjugated Molecular Films, J. Polym. Sci. B 41, 2529 (2003).
[12] N. Koch, Organic Electronic Devices and Their Functional Interfaces, Chem. Phys. Chem. 8, 1438 (2007).

[13] K. Rüdenberg, Quantum Mechanics of Mobile Electrons in Conjugated Bond Systems. III. Topological Matrix as Generatrix of Bond Orders, J. Chem. Phys. 34, 1884 (1961).

[14] R. B. Mallion and D. H. Rouvray, The Golden Jubilee of the Coulson-Rushbrooke Pairing Theorem, J. Math. Chem. 5, 1 (1990).

[15] M. Liu, M. Liu, Z. Zha, J. Pan, X. Qiu, T. Li, J. Wang, Y. Zheng, and D. Zhong, Thermally Induced Transformation of Nonhexagonal Carbon Rings in Graphene-like Nanoribbons, J. Phys. Chem. C 122, 9586 (2018).

[16] J. Hieulle, E. Carbonell-Sanrom, M. Vilas-Varela, A. Garcia-Lekue, E. Guitin, D. Peña, and J. I. Pascual, On-surface Route for Producing Planar Nanographenes with Azulene Moieties, Nano Lett. 18, 418 (2018).

[17] A. Shiotari, T. Nakae, K. Iwata, S. Mori, T. Okujima, H. Uno, H. Sakaguchi, and Y. Sugimoto, Strain-induced Skeletal Rearrangement of a Polycyclic Aromatic Hydrocarbon on a Copper Surface, Nat. Commun. 8, 16089 (2017).

[18] S. Mishra, T. G. Lohr, C. A. Pignedoli, J. Liu, R. Berger, J. I. Urgel, K. Müllen, X. Feng, P. Ruffieux, and R. Fasel, Tailoring Bond Topologies in Open-Shell Graphene Nanostructures, ACS Nano, 12, 11917 (2018).

[19] H. Xin and X. Gao, Application of Azulene in Constructing Organic Optoelectronic Materials: New Tricks for an Old Dog, Chem. Plus Chem. 82, 945 (2017).

[20] Y. Yamaguchi, M. Takubo, K. Ogawa, K.-i. Nakayama, T. Koganezawa, and H. Katagiri, Terazulene Isomers: Polarity Change of OFETs through Molecular Orbital Distribution Contrast, J. Am. Chem. Soc. 138, 11335 (2016).

[21] P. Y. Huang, C. S. Ruiz-Vargas, A. M. van der Zande, W. S. Whitney, M. P. Levendorf, J. W. Kevek, S. Garg, J. S. Alden, C. J. Hustedt, Y. Zhu, J. Park, P. L. McEuen, and D. A. Muller, Grains and Grain Boundaries in Single-Layer Graphene Atomic Patchwork Quilts, Nature (London) 469, 389 (2011).

[22] F. Banhart, J. Kotakoski, and A. V. Krasheninnikov, Structural Defects in Graphene, ACS Nano 5, 26 (2011).

[23] J. Červenka and C. F. J. Flipse, Structural and Electronic Properties of Grain Boundaries in Graphite: Planes of Periodically Distributed Point Defects, Phys. Rev. B 79, 195429 (2009).

[24] N. M. R. Peres, F. Guinea, and A. H. C. Neto, Electronic Properties of Disordered Two-Dimensional Carbon, Phys. Rev. B 73, 125411 (2006).

[25] R. Grantab, V. B. Shenoy, and R. S. Ruoff, Anomalous Strength Characteristics of Tilt Grain Boundaries in Graphene, Science 330, 946 (2010).

[26] Y. Wei, J. Wu, H. Yin, X. Shi, R. Yang, and M. Dresselhaus, The Nature of Strength Enhancement and Weakening by Pentagon-Heptagon Defects in Graphene, Nat. Mat. 11, 759 (2012).

[27] J. Červenka, M. I. Katsnelson, and C. F. J. Flipse, Room-Temperature Ferromagnetism in Graphite Driven by Two-dimensional Networks of Point Defects, Nat. Phys. 5, 840 (2009). 
[28] S. Malola, H. Häkkinen, and P. Koskinen, Structural, Chemical, and Dynamical Trends in Graphene Grain Boundaries, Phys. Rev. B 81, 165447 (2010).

[29] K. Müller, A. P. Seitsonen, T. Brugger, J. Westover, T. Greber, T. Jung, and A. Kara, Electronic Structure of an Organic/Metal Interface: Pentacene/Cu(110), J. Phys. Chem. C 116, 23465 (2012).

[30] F. S. Tautz, Structure and Bonding of Large Aromatic Molecules on Noble Metal Surfaces: The Example of PTCDA, Prog. Surf. Sci. 82, 479 (2007).

[31] E. Umbach, Characterization of Organic Overlayers on Well-Defined Substrates, Prog. Surf. Sci. 35, 113 (1990).

[32] K. Seki, N. Hayashi, H. Oji, E. Ito, Y. Ouchi, and H. Ishii, Electronic Structure of Organic/Metal Interfaces, Thin Solid Films 393, 298 (2001).

[33] The Molecule-Metal Interface, edited by N. Koch, N. Ueno, and A. T. S. Wee (Wiley-VCH, New York, 2013).

[34] T. Yamada, M. Shibuta, Y. Ami, Y. Takano, A. Nonaka, K. Miyakubo, and T. Munakata, Novel Growth of Naphthalene Overlayer on Cu(111) Studied by STM, LEED, and 2PPE, J. Phys. Chem. C 114, 13334 (2010).

[35] L.-J. Wan and K. Itaya, In Situ Scanning Tunneling Microscopy of Benzene, Naphthalene, and Anthracene Adsorbed on $\mathrm{Cu}(111)$ in Solution, Langmuir 13, 7173 (1997).

[36] R. Forker, J. Peuker, M. Meissner, F. Sojka, T. Ueba, T. Yamada, H. S. Kato, T. Munakata, and T. Fritz, The Complex Polymorphism and Thermodynamic Behavior of a Seemingly Simple System: Naphthalene on $\mathrm{Cu}(111)$, Langmuir 30, 14163 (2014).

[37] S. Lukas, S. Vollmer, G. Witte, and C. Wöll, Adsorption of Acenes on Flat and Vicinal Cu(111) Surfaces: Step Induced Formation of Lateral Order, J. Chem. Phys. 114, 10123 (2001).

[38] H. Wang, G. Dutton, and X. Y. Zhu, Electronic Structure at Organic/Metal Interfaces: Naphthalene/Cu(111), J. Phys. Chem. B 104, 10332 (2000).

[39] H. Tetlow, J. P. de Boer, I. J. Ford, D. D. Vvedensky, J. Coraux, and L. Kantorovich, Growth of Epitaxial Graphene: Theory and Experiment, Phys. Rep. 542, 195 (2014).

[40] L. Gao, J. R. Guest, and N. P. Guisinger, Epitaxial Graphene on $\mathrm{Cu}(111)$, Nano Lett. 10, 3512 (2010).

[41] X. Li, C. W. Magnuson, A. Venugopal, J. An, J. W. Suk, B. Han, M. Borysiak, W. Cai, A. Velamakanni, Y. Zhu, L. Fu, E. M. Vogel, E. Voelkl, L. Colombo, and R.S. Ruoff, Graphene Films with Large Domain Size by a Two-Step Chemical Vapor Deposition Process, Nano Lett. 10, 4328 (2010).

[42] Q. Fan, J. M. Gottfried, and J. Zhu, Surface-Catalyzed C-C Covalent Coupling Strategies Toward the Synthesis of LowDimensional Carbon-Based Nanostructures, Acc. Chem. Res. 48, 2484 (2015).

[43] M. Murai, S. Iba, H. Ota, and K. Takai, Azulene-fused Linear Polycyclic Aromatic Hydrocarbons with Small Bandgap, High Stability, and Reversible Stimuli Responsiveness, Org. Lett. 19, 5585 (2017).

[44] See Supplemental Material at http://link.aps.org/ supplemental/10.1103/PhysRevX.9.011030 for details of the coverage determination, the NIXSW, TPD, and NEX-
AFS data treatment, as well as additional XPS, STM, and nc-AFM data, and further details of the DFT calculations.

[45] F. C. Bocquet, G. Mercurio, M. Franke, G. van Straaten, S. Wei, S. Soubatch, C. Kumpf, and F. S. Tautz, Torricelli: A Software to Determine Atomic Spatial Distributions from Normal Incidence X-ray Standing Wave Data, Comput. Phys. Commun. 235, 502 (2019).

[46] S. G. Hessey and R. G. Jones, Line-of-Sight Mass Spectrometry: Principles and Practice, Surf. Interface Anal. 47, 587 (2015).

[47] I. Horcas, R. Fernandez, J. M. Gomez-Rodriguez, J. Colchero, J. Gomez-Herrero, and A. M. Baro, WSXM: A Software for Scanning Probe Microscopy and a Tool for Nanotechnology, Rev. Sci. Instrum. 78, 013705 (2007).

[48] G. Kresse and J. Hafner, Ab Initio Molecular Dynamics for Liquid Metals, Phys. Rev. B 47, 558 (1993).

[49] G. Kresse and J. Hafner, Ab Initio Molecular-Dynamics Simulation of the Liquid-Metal-Amorphous-Semiconductor Transition in Germanium, Phys. Rev. B 49, 14251 (1994).

[50] G. Kresse and J. Furthmüller, Efficiency of Ab-Initio Total Energy Calculations for Metals and Semiconductors Using a Plane-Wave Basis Set, Comput. Mater. Sci. 6, 15 (1996).

[51] G. Kresse and J. Furthmüller, Efficient Iterative Schemes for Ab Initio Total-Energy Calculations Using a Plane-Wave Basis Set, Phys. Rev. B 54, 11169 (1996).

[52] J. P. Perdew, K. Burke, and M. Ernzerhof, Generalized Gradient Approximation Made Simple, Phys. Rev. Lett. 77, 3865 (1996).

[53] S. Grimme, J. Antony, S. Ehrlich, and H. Krieg, A Consistent and Accurate Ab Initio Parametrization of Density Functional Dispersion Correction (DFT-D) for the 94 Elements $\mathrm{H}-\mathrm{Pu}$, J. Chem. Phys. 132, 154104 (2010).

[54] P. E. Blöchl, Projector Augmented-Wave Method, Phys. Rev. B 50, 17953 (1994).

[55] G. Kresse and D. Joubert, From Ultrasoft Pseudopotentials to the Projector Augmented-Wave Method, Phys. Rev. B 59, 1758 (1999).

[56] S. J. Clark, M. D. Segall, C. J. Pickard, P. J. Hasnip, M. I. J. Probert, K. Refson, and M. C. Payne, First Principles Methods Using CASTEP, Z. Kristallogr. Cryst. Mater. 220, 567 (2009).

[57] M. Teruyasu, T. Isao, G. Shang-Peng, and J. P. Chris, FirstPrinciples Calculation of Spectral Features, Chemical Shift and Absolute Threshold of ELNES and XANES Using a Plane Wave Pseudopotential Method, J. Phys. Condens. Matter 21, 104204 (2009).

[58] L. Triguero, L. G. M. Pettersson, and H. Ågren, Calculations of Near-Edge X-Ray-Absorption Spectra of Gas-Phase and Chemisorbed Molecules by Means of Density-Functional and Transition-Potential Theory, Phys. Rev. B 58, 8097 (1998).

[59] M. Klues, K. Hermann, and G. Witte, Analysis of the NearEdge X-Ray-Absorption Fine-Structure of Anthracene: A Combined Theoretical and Experimental Study, J. Chem. Phys. 140, 014302 (2014).

[60] K. Diller, R. J. Maurer, M. Müller, and K. Reuter, Interpretation of X-Ray Absorption Spectroscopy in the Presence of Surface Hybridization, J. Chem. Phys. 146, 214701 (2017). 
[61] R. J. Maurer and K. Reuter, Excited-State Potential-Energy Surfaces of Metal-Adsorbed Organic Molecules from Linear Expansion $\Delta$-Self-Consistent Field Density-Functional Theory $(\triangle S C F-D F T)$, J. Chem. Phys. 139, 014708 (2013).

[62] D. P. Woodruff, Normal Incidence X-Ray Standing Wave Determination of Adsorbate Structures, Prog. Surf. Sci. 57, 1 (1998)

[63] D. P. Woodruff, Surface Structure Determination Using X-Ray Standing Waves, Rep. Prog. Phys. 68, 743 (2005).

[64] J. Zegenhagen, Surface Structure Determination with X-Ray Standing Waves, Surf. Sci. Rep. 18, 202 (1993).

[65] The issue of unrealistically high coherent fractions, recently observed in several measurements performed at the I09 beam line, did not occur in our case. This is possibly due to the moderate maximum count rates we allowed during data acquisition, in order to avoid a possible nonlinearity of the multichannel plate detector.

[66] B. Stadtmüller, S. Schröder, and C. Kumpf, Heteromolecular Metalorganic Interfaces: Electronic and Structural Fingerprints of Chemical Bonding, J. Electron Spectrosc. Relat. Phenom. 204, 80 (2015).

[67] D. R. Lide, CRC Handbook of Chemistry and Physics, 90th ed. (CRC Press, Boca Raton, FL, 2010).

[68] K. Christmann, Introduction to Surface Physical Chemistry (Steinkopff Verlag, Springer-Verlag, Darmstadt, New York, 1991).

[69] A. M. de Jong and J. W. Niemantsverdriet, Thermal Desorption Analysis: Comparative Test of Ten Commonly Applied Procedures, Surf. Sci. 233, 355 (1990).

[70] D. L. S. Nieskens, A. P. van Bavel, and J. W. Niemantsverdriet, The Analysis of Temperature Programmed Desorption Experiments of Systems with Lateral Interactions; Implications of the Compensation Effect, Surf. Sci. 546, 159 (2003).

[71] G. Witte, S. Lukas, P. S. Bagus, and C. Wöll, Vacuum Level Alignment at Organic/Metal Junctions: Cushion Effect and the Interface Dipole, Appl. Phys. Lett. 87, 263502 (2005).

[72] I. Fernandez-Torrente, S. Monturet, K. J. Franke, J. Fraxedas, N. Lorente, and J. I. Pascual, Long-Range Repulsive Interaction between Molecules on a Metal Surface Induced by Charge Transfer, Phys. Rev. Lett. 99, 176103 (2007).

[73] S. Lukas, G. Witte, and C. Wöll, Novel Mechanism for Molecular Self-Assembly on Metal Substrates: Unidirectional Rows of Pentacene on Cu(110) Produced by a Substrate-Mediated Repulsion, Phys. Rev. Lett. 88, 028301 (2001).

[74] G. Tomba, M. Stengel, W. D. Schneider, A. Baldereschi, and A. De Vita, Supramolecular Self-Assembly Driven by Electrostatic Repulsion: The 1D Aggregation of Rubrene Pentagons on Aul11, ACS Nano 4, 7545 (2010).

[75] E. Bauer, F. Bonczek, H. Poppa, and G. Todd, Thermal Desorption of Metals from Tungsten Single Crystal Surfaces, Surf. Sci. 53, 87 (1975).

[76] D. A. King, Thermal Desorption from Metal Surfaces: A Review, Surf. Sci. 47, 384 (1975).

[77] S. L. Tait, Z. Dohnálek, C. T. Campbell, and B. D. Kay, n-Alkanes on MgO(100). I. Coverage-Dependent
Desorption Kinetics of n-Butane, J. Chem. Phys. 122, 164707 (2005).

[78] D. P. Chong, Density Functional Theory Study on the Electron Spectra of Naphthalene and Azulene Vapours, Can. J. Chem. 88, 787 (2010).

[79] S. Doniach and M. Šunjić, Many-Electron Singularity in X-Ray Photoemission and X-Ray Line Spectra from Metals, J. Phys. C 3, 285 (1970).

[80] W. Zhao, W. Wei, and J. M. White, Two-Photon Photoemission Spectroscopy: Naphthalene on Cu(111), Surf. Sci. 547, 374 (2003).

[81] F. Bischoff, K. Seufert, W. Auwärter, S. Joshi, S. Vijayaraghavan, D. Écija, K. Diller, A. C. Papageorgiou, S. Fischer, F. Allegretti, D. A. Duncan, F. Klappenberger, F. Blobner, R. Han, and J. V. Barth, How Surface Bonding and Repulsive Interactions Cause Phase Transformations: Ordering of a Prototype Macrocyclic Compound on Ag (111), ACS Nano 7, 3139 (2013).

[82] J. Tersoff and D. R. Hamann, Theory of the Scanning Tunneling Microscope, Phys. Rev. B 31, 805 (1985).

[83] B. Schuler, W. Liu, A. Tkatchenko, N. Moll, G. Meyer, A. Mistry, D. Fox, and L. Gross, Adsorption Geometry Determination of Single Molecules by Atomic Force Microscopy, Phys. Rev. Lett. 111, 106103 (2013).

[84] F. Mohn, L. Gross, N. Moll, and G. Meyer, Imaging the Charge Distribution within a Single Molecule, Nat. Nanotechnol. 7, 227 (2012).

[85] L. Gross, F. Mohn, P. Liljeroth, J. Repp, F. J. Giessibl, and G. Meyer, Measuring the Charge State of an Adatom with Noncontact Atomic Force Microscopy, Science 324, 1428 (2009).

[86] B. Schuler, S.-X. Liu, Y. Geng, S. Decurtins, G. Meyer, and L. Gross, Contrast Formation in Kelvin Probe Force Microscopy of Single p-conjugated Molecules, Nano Lett. 14, 3342 (2014).

[87] J. Wellendorff, T. L. Silbaugh, D. Garcia-Pintos, J. K. Nørskov, T. Bligaard, F. Studt, and C. T. Campbell, A Benchmark Database for Adsorption Bond Energies to Transition Metal Surfaces and Comparison to Selected DFT Functionals, Surf. Sci. 640, 36 (2015).

[88] R. J. Maurer, V. G. Ruiz, and A. Tkatchenko, Many-body Dispersion Effects in the Binding of Adsorbates on Metal Surfaces, J. Chem. Phys. 143, 102808 (2015).

[89] R. J. Maurer, W. Liu, I. Poltavsky, T. Stecher, H. Oberhofer, K. Reuter, and A. Tkatchenko, Thermal and Electronic Fluctuations of Flexible Adsorbed Molecules: Azobenzene on Ag(111), Phys. Rev. Lett. 116, 146101 (2016).

[90] R. F. W. Bader, Atoms in Molecules-A Quantum Theory (Oxford University Press, Oxford, 1990).

[91] E. Hückel, Quantentheoretische Beiträge Zum Problem der Aromatischen und Ungesättigten Verbindungen. III, Z. Phys. 76, 628 (1932).

[92] E. Clar, The Aromatic Sextet, 2nd ed. (John Wiley \& Sons, London, New York, Sydney, Toronto, 1972).

[93] A. A. Frost and B. Musulin, A Mnemonic Device for Molecular Orbital Energies, J. Chem. Phys. 21, 572 (1953).

[94] C. A. Coulson and G. S. Rushbrooke, Note on the Method of Molecular Orbitals, J. Phys. Condens. Matter 36, 193 (1940). 
[95] F. L. Hirshfeld, Bonded-Atom Fragments for Describing Molecular Charge Densities, Theor. Chim. Acta 44, 129 (1977).

[96] H. Vita, S. Böttcher, K. Horn, E. N. Voloshina, R. E. Ovcharenko, T. Kampen, A. Thissen, and Y. S. Dedkov, Understanding the Origin of Band Gap Formation in
Graphene on Metals: Graphene on $\mathrm{Cu} / \mathrm{Ir}(111)$, Sci. Rep. 4, 5704 (2014).

[97] H. S. Moon, J. H. Lee, S. Kwon, I. T. Kim, and S. G. Lee, Mechanisms of Na Adsorption on Graphene and Graphene Oxide: Density Functional Theory Approach, Carbon Lett. 16, 116 (2015). 